\title{
USO DELLA CANZONE NELLA CLASSE DI LINGUA E CULTURA PER SVILUPPARE LA COMPETENZA SOCIOCULTURALE
}

\author{
Hermis Tolentino Quiñones ${ }^{1}$
}

\section{LA CANZONE NELLA CLASSE DI LINGUA E CULTURA}

Nel mondo, la lingua italiana viene studiata sempre di più, così lo dimostrano i dati del Ministero degli Affari Esteri e della Cooperazione Internazionale; in effetti, la lingua di Dante viene diffusa e trasmessa in tutto il mondo da diverse associazioni: Università, scuole pubbliche e private, Istituti Italiani di Cultura, ecc., raggiungendo i 2.145.093 studenti in 115 Paesi, fra il 2016 e il 2017. Anche in Perù c'è stata una crescita dei numeri di iscritti ai corsi di lingua e cultura italiana, raggiungendo il numero di 17.137, arrivando così a occupare il diciannovesimo posto a livello mondiale (Ministero degli Affari Esteri e della Cooperazione Internazionale, 2018). Perciò, di fronte all'incremento degli amanti della lingua e la cultura italiana, è necessario che le associazioni, attraverso i loro docenti, possano offrire una didattica che vada d'accordo con i bisogni e le necessità linguistiche degli studenti e che possa mantenere la motivazione durante tutto il percorso formativo.

Per raggiungere l'obiettivo di mantenere la motivazione sorge sicuramente la domanda "come ci si aspetta che gli studenti imparino una seconda lingua (L2) o una lingua straniera (LS)?”. Nel Quadro Comune Europeo di Riferimento per le lingue (QCER, 2002) si cerca di dare una risposta, indicando che questo è possibile ascoltando la radio, le registrazioni, guardando e ascoltando la televisione, ecc., esponendo l'apprendente a un imput (orale) il più autentico possibile. È ovvio che qui si parla di sviluppare la comprensione orale, cioè, si tratta di sviluppare l'abitudine dell'ascolto attraverso un lavoro sistematico e pianificato per, poi, interagire facendo uso della lingua parlata: prima ascoltata e dopo parlata (Sánchez et al., 2019). Esporre lo studente a un input il più autentico e comprensibile possibile, oggi, non è tanto difficile: lo sviluppo della tecnologia e la creazione di strumenti come l'iPod, il telefono intelligente, il riproduttore $M P 3$, ecc., rendono possibile agli studenti ascoltare la radio, guardare videoclip, sentire della musica, ecc. (Mamani, 2018).

Allo scopo di esporre lo studente a un input autentico e orale, e per mantenere la motivazione, si può fare uso della musica in quanto il testo musicale è un testo autentico ricco di elementi linguistici e culturali, capace di coniugare stimoli legati allo sviluppo della competenza lessicale, grammaticale, fonologica, sintattica e testuale. Inoltre, è un testo che incentiva lo sviluppo della competenza socioculturale permettendo l'analisi di elementi legati alla varietà linguistica e perché fa riferimento al contesto socioculturale e interculturale dello studente (Mauroni, 2011). Quindi, la musica senza dubbio può essere di grande aiuto nella didattica delle lingue moderne.

Comunemente si dice che la musica è il linguaggio universale, e quindi, nella lezione di lingua e cultura è necessario il suo uso in quanto è uno strumento della comunicazione verbale intrapersonale, è la forma tangibile per esprimere i nostri pensieri, sentimenti, conoscenze, ecc. (Sánchez et al., 2019). Inoltre, la musica, come linguaggio, è capace di comunicare, evocare e rafforzare le diverse emozioni dell'individuo (Custodio, Cano-

\footnotetext{
${ }^{1}$ Universidad César Vallejo, Víctor Larco Herrera (Trujillo), Perú. ORCID: https:/ / orcid.org/0000-0002-
} 9819-1655. 
Campos, 2017). Infine, la musica fa parte della quotidianità di ogni individuo, essa è usata nei film, negli annunci, alla radio, alla televisione, addirittura nei luoghi pubblici: autobus, bar, ecc. (Mamani, 2018). Quindi, far ascoltare musica in classe non è altro che portare la quotidianità dello studente nella lezione di lingua e cultura e saper sfruttare i benefici che se ne possono trarre; in questo senso, è importante considerare che l'attività di ascolto è volontaria e ha come scopo soddisfare i bisogni dell'ascoltatore (Sánchez et al., 2019), obiettivi che possono essere linguistici, comunicativi e culturali, e perché no, anche di tipo ludico, legati al "piacere", perché ascoltare una canzone può servire semplicemente per sentirsi bene e rilassarsi.

D'accordo con Mauroni (2011), in una prospettiva di insegnamento/apprendimento (in questo caso della lingua italiana come L2 o LS) centrato sull'approccio umanisticoaffettivo, che pone lo studente al centro del processo di apprendimento, è necessario che lo studente sia autonomo e responsabile del proprio processo di apprendimento e la canzone è uno strumento prezioso in quanto è legato alla motivazione dell'apprendente.

\subsection{Benefici dell'uso della musica in classe}

I benefici dell'uso della canzone nell'insegnamento/apprendimento di una lingua straniera come lo è l'italiano possono essere molti, sia dal punto di vista della motivazione che dal punto di vista linguistico e interculturale. Caon (n.d.) sottolinea che l'uso della canzone in classe permette all'insegnante di intervenire nell'apprendente su due aspetti: il primo, sui processi consci e, il secondo, sui processi inconsci, tutti e due nello stesso tempo, integrando in questo modo gli stimoli cognitivi e gli stimoli emotivi ed affettivi che permettono di avere un apprendimento più profondo e duraturo. In questo senso, si può dire che, dal punto di vista cognitivo, nell'ascoltare una canzone si attivano una serie di processi all'interno della mente dello studente, tra questi l'elaborazione, la trasformazione, l'immagazzinamento e il recupero dell'informazione sonora che si trova all'interno dell'input musicale (Allonello, 2005). Inoltre, l'uso della canzone nella lezione di lingua e cultura non solo stimola l'apprendimento linguistico ma anche permette di rafforzare, se non costruire, la consapevolezza fonologica e permette uno sviluppo sempre maggiore dell'abilità di ascolto (Beozzo, 2013). Inoltre, dal punto di vista emotivo, la musica ha la capacità di scatenare una serie di emozioni, positive o negative che siano (Custodio, Cano-Campos, 2017).

Caon (n.d.) elenca una serie di vantaggi nell'utilizzo della canzone nell'insegnamento/apprendimento di una lingua straniera:

- facilita l'attivazione di una motivazione basata sul piacere. In questo senso, si può dire che non esiste nessuna persona a cui non piaccia ascoltare musica, anzi, la si ascolta volentieri anche ripetutamente. Inoltre, il ritmo chiaro e le melodie semplici (ad es. della musica pop) fanno sì che il testo sia di facile memorizzazione (Balboni, 2018);

- è didattizzabile sotto molti aspetti: in quanto materiale autentico dà la possibilità di lavorare sull'aspetto fonetico/fonologico, lessicale, grammaticale, culturale e interculturale;

- favorisce la memorizzazione perché la canzone viene ripetuta molte volte senza che questo diventi noioso (a patto che la canzone piaccia allo studente). In questo senso, Balboni (2018) osserva che la brevità dei testi della canzone permette di costruire intorno a essi delle unità di acquisizione compatte;

- è uno stimolo polisemico, il quale può essere mono o multisettoriale, permettendo l'attivazione di tutti e due gli emisferi cerebrali (dal destro al sinistro) per sviluppare diverse abilità: ascolto, lettura, scrittura e parlato. 


\subsection{Punti critici della canzone}

Sebbene la canzone/musica abbia diversi vantaggi, dal punto di vista cognitivo ed emotivo/affettivo, vi sono anche alcuni punti critici che devono essere affrontati e risolti da un docente attento nel momento di didattizzare il testo autentico:

- in generale, la comprensione auditiva richiede un'intensa attività di analisi e sintesi, induzione e deduzione, astrazione e concretizzazione (Sánchez et al., 2019). Nell'ascoltare, lo studente elabora un processo linguistico che comprende l'identificare dei suoni velocemente e il trattenere l'informazione in un determinato contesto, perciò è importante elaborare delle attività di preascolto;

- la musica comprende una relazione di integrazione sociale che oltrepassa le preferenze individuali; perciò, ascoltare una stessa canzone può risultare piacevole per alcuni e meno piacevole per altri. Quindi, l'ascolto di una canzone permette a ogni apprendente di darne un'interpretazione unica e diversa (Oliveira, Silva, 2018). In questo senso, è compito dell'insegnante saper guidare lo studente ad esprimere le sue idee e le sue emozioni;

- essendo la canzone un testo autentico potrebbe presentare un lessico ed espressioni difficili da comprendere. Anche la presenza di elementi grammaticali, come, per esempio, i tempi e i modi verbali, possono costituire una difficoltà nella comprensione del testo. Anche dal punto di vista della pronuncia si potrebbero presentare delle difficoltà, ad esempio una falsa intonazione.

\section{LA SCELTA DELLA CANZONE}

La canzone è un testo "misto" formato da parole e musica e, come tale, nella sua scelta per la didattizzazione bisogna considerare alcuni aspetti, tra cui la tipologia dei destinatari, i loro bisogni comunicativi, linguistici e culturali, la loro provenienza linguistica, il loro livello di conoscenza della lingua che stanno imparando, l'approccio e il metodo che si usa o al quale gli studenti sono abituati, gli stili cognitivi e anche i tipi di intelligenza degli allievi, oltre che le caratteristiche linguistiche (lessicali, morfosintattiche, ecc.) e stilistiche del il testo della canzone (Begotti, n.d.). Ł̀ anche importante considerare che la canzone può essere presentata in formato videoclip, audio e testo. Begotti (n.d.) elenca alcuni elementi da considerare nella didattizzazione della canzone:

- la scelta del materiale autentico. Il testo può presentare elementi linguistici diversi rispetto a quelli indicati nel sillabo previsto per il livello di apprendimento della lingua degli studenti. In questo caso è importante che il docente sia in grado di anticipare le possibili difficoltà che essi potrebbero incontrare;

- la corrispondenza con gli obiettivi didattici proposti. È importante avere chiaro se il testo che si didattizzerà ha come obiettivo un percorso guidato oppure se viene proposto per l'autoapprendimento. È altrettanto importante considerare le preconoscenze degli studenti per raggiungere gli obiettivi proposti;

- la presenza di varietà linguistica. Come si è più sopra accennato, la canzone è un testo autentico e come tale può presentare una serie di varietà linguistiche di cui il docente deve esserne consapevole per creare delle attività didattiche che rendano il materiale didattico comprensibile e non attivino il filtro affettivo;

- la qualità del sonoro e del visivo. Una canzone che si ascolta male per problemi tecnici, anche se bella sarà demotivante e non si raggiungeranno gli obiettivi proposti. Bisogna anche considerare che la canzone può essere presentata come materiale audiovisivo 
in quanto fa uso del sonoro, dell'immagine e della scrittura, e a questo proposito Torresan (n.d.) considera che i materiali di questo genere integrano diversi codici proprio come succede nella quotidianità delle persone.

\section{MODELLO OPERATIVO E TECNICHE DIDATTICHE PER DIDIDATTIZZARE UNA CANZONE}

Nell'ambito della glottodidattica moderna della lingua italiana sono stati proposti diversi modelli operativi, tra questi è possibile citare quello basato sull'Unità di lavoro di Diadori, l'Unità didattica centrata sul testo di Vedovelli e l'Unità didattica di Freddi. Per le attività presentate in questo studio è stata adottato il modello di Unità Didattica di Freddi ripresa da Balboni.

Come è noto, l'Unità Didattica proposta da Freddi si compone di sei parti: la motivazione, la globalità, l'analisi, la sintesi, la riflessione e il controllo.

Si tratta di un modello lineare e strutturato da intendersi come «tranche linguisticocomunicativa complessa, realizzata mettendo insieme eventi, atti, espressioni, strutture linguistiche legati ad un contesto situazionale» (Balboni, 2002: 103) della durata di 6-10 ore che Balboni suggerisce di adattare ed integrare con il modello più flessibile e modulare dell'Unità di Apprendimento, di più breve e di durata variabile (corrispondente ad una unità di "lezione"), organizzato in modo non lineare, ma a "rete", con riprese e adattamenti ciclici, così da permettere una maggiore dinamicità e adattabilità ai bisogni linguistici, comunicativi e culturali degli studenti. Si intende quindi come Unità di Apprendimento il singolo incontro in classe che può avere una durata di 30 minuti o un massimo di due ore con obiettivi specifici da raggiungere. L'Unità di Apprendimento segue la struttura dell'Unità Didattica, ma non ne comprende necessariamente tutte le fasi che possono ridursi o alternarsi in funzione degli obiettivi che si pone.

Nel progettare e proporre una Unità di Apprendimento (UdA) è importante valutare lo stile di apprendimento degli studenti, considerato come un insieme di strategie a livello cognitivo che lo studente mette in atto per poter apprendere (Pichiassi, 2009), nel nostro caso, una lingua. Da questo punto di vista, la canzone si adatta con facilità a ogni tipo di stile di apprendimento ma il docente deve saper scegliere l'approccio e le tecniche didattiche adatte alla classe.

\section{Metodologia}

Il presente studio ha seguito la metodologia della ricerca-azione che «es una forma de investigación que permite vincular el estudio de los problemas en un contexto determinado con programas de acción social, de manera que se logren de forma simultánea conocimientos y cambios sociales» ${ }^{2}$ (Vidal, Rivera, 2007: s.p.). Nello specifico, nell'ambito della glottodidattica, il docente deve pianificare e stabilire una serie di passaggi in modo articolato per intervenire nella sua prassi didattica e raggiungere gli obiettivi proposti (Coonan, n.d.). Ed è in questo senso che il presente lavoro ha come obiettivo dimostrare che l'uso della canzone nei corsi di lingua e cultura italiana non solo permette di sviluppare la competenza linguistica, sociolinguistica, pragmatica e metalinguistica (Novello, n.d.), ma serve anche a riflettere sul proprio vissuto e su quello che accade nella società.

\footnotetext{
${ }^{2}$ La ricerca-azione «è una forma di ricerca che collega lo studio dei problemi in un dato contesto con programmi di azione sociale, in modo che la conoscenza e il cambiamento sociale siano raggiunti simultaneamente».
} 
(C) Italiano LinguaDue 2. 2021. H. Tolentino Quiñones, Uso della canzone nella classe di lingua e cultura per sviluppare la competenza socioculturale

\section{APPLICAZIONE DELLE UNITÀ DI APPRENDIMENTO ${ }^{3}$}

\subsection{Struttura delle Unità di Apprendimento}

La struttura proposta è quella dell'UdA (motivazione, globalità, analisi-riflessione e sintesi). Ogni unità si apre con una specie di copertina con l'immagine dell'artista o del gruppo e i dati più importanti che li riguardano. Segue una seconda parte che consiste nel presentare una serie di immagini per attivare le conoscenze pregresse e attivare l'emisfero destro del cervello. Queste attività corrispondono alla fase della motivazione e del preascolto.

La fase della comprensione globale prevede di far vedere agli studenti il videoclip della canzone e far svolgere loro alcune attività quali, ad esempio, mettere in ordine delle parole, completare il ritornello e rispondere con vero/falso, cui segue un cloze. Nella fase di analisi, per guidare la comprensione approfondita, sono proposte attività sul lessico e su alcuni argomenti grammaticali (ma come riconoscimento e ripasso). Poi vengono poste delle domande a cui rispondere per scritto.

Per svolgere la fase della riflessione e sintesi si propongono attività che invitano gli studenti a interpretare il testo della canzone, fare riassunti, esprimere opinioni, attività di transcodificazione e attività di produzione collegando il messaggio del testo alla propria esperienza.

\subsection{Gruppi di applicazione delle Unità di Apprendimento.}

L'UdA è stata sperimentata con tre gruppi di studenti.

Il primo gruppo era composto da 10 studenti (sei femmine e quattro maschi), fra i 22 e 35 anni, nel corso di lingua e cultura italiana di livello Medio - B1 presso l'Istituto Italiano di Cultura di Lima. La canzone, Ballata dell'amore cieco ${ }^{4}$, è stata usata l'ultimo giorno di lezione, per un ripasso prima dell'esame finale (ogni mese si è svolto un test scritto e orale per passare poi alla fase successiva). Le lezioni si sono svolte a distanza usando la piattaforma Classroom di Google per le attività asincrone, per le attività sincrone si è utilizzato Zoom.

Il secondo gruppo era formato da studenti dell'Università Nacional de Educaciòn Enrique Guzmán y Valle. Il gruppo era composto da 16 studenti (13 femmine e tre maschi), l'età media è 21 anni. È importante sottolineare che questo gruppo ha studiato la lingua italiana durante tutto il semestre accademico e che l'UdA si è svolta nella quarta settimana del corso. La canzone I cento passi è stata usata dopo la morte di due studenti avvenuta durante una manifestazione avvenuta in Perù contro l'allora presidente della Repubblica; l'obiettivo è stato quindi far riflettere gli studenti da una parte su quello che stava succedendo in Perù e, dall'altra, sull'omicidio di Peppino Impastato, giovane attivista impegnato in Italia nella lotta alla mafia. Per le attività asincrone si è usato Moodle, invece per le attività sincrone si è fatto ricorso a Google Meet. In una lezione successiva si è lavorato sulla scena del film I cento passi in cui Peppino raggiunge la casa del boss della mafia. Si è presentata anche la canzone Che fantastica storia è la vita con l'obiettivo di far riflettere gli studenti su aspetti relativi ai loro interessi e obiettivi personali. Gli studenti sono stati invitati a costruire una specie di album virtuale dove ognuno ha scritto la propria presentazione e postato una sua fotografia.

\footnotetext{
${ }^{3}$ Le Unità di Apprendimento sono riportate nell'appendice.
}

${ }^{4}$ https://www.youtube.com/watch?v=HXE8waG_X-4. 
Anche il terzo gruppo di studenti apparteneva all’Università Enrique Guzmán y Valle ed era formato da 14 studenti (10 femmine e quattro maschi) con caratteristiche molto simili al gruppo precedente, con la differenza che erano apprendenti a livello B1. Per l'UdA rivolta a questi studenti si è usata la canzone Pensa di Francesco Moro anche per riflettere sulla morte dei due studenti alla manifestazione contro il colpo di stato effettuato in Perù.

Si sono utilizzate anche altre App per rendere le attività più motivanti: Wordwall, Playposit, LearningApps, Moro, Site e YouTube. Gli studenti hanno ricevuto un documento in pdf accompagnato da attività adattate per il suo uso online.

\subsection{Applicazioni delle unità di apprendimento e riflessioni}

Per il lavoro della sperimentazione e per riflettere sull'applicazione delle UdA si è fatto uso di un'agenda, nella quale si sono annotati le reazioni degli studenti e il modo di come si è svolta la lezione. I dati emersi sono riportati in tre momenti: prima, durante e dopo l'applicazione dell'UdA. È importante sottolineare che, per avere una maggiore oggettività, gli appunti sono stati elaborati dopo la visione della registrazione delle lezioni.

\subsubsection{Ballata dell'amore cieco di Fabrizio di André}

La canzone Ballata dell'amore cieco di Fabrizio di André racconta la storia di un uomo che per amore è capace di uccidere la madre e di togliersi la vita su richiesta della donna amata. Tuttavia, egli muore felice mentre lei rimane in vita ma presa da sgomento. Il materiale didattico è pensato per un livello B1 del QCER e ha come obiettivo riflettere sull'amore e la felicità. Dal punto di vista didattico-linguistico gli obiettivi sono: arricchire il bagaglio lessicale e ripassare la forma del passato remoto.

Prima: il docente comunica agli studenti che ascolteranno una canzone. Alcuni di loro, principalmente le donne, dicono: "che bello", "davvero?", e incuriositi iniziano a chiedere di quale canzone e di quale cantante si tratti. Subito, approfittando della loro curiosità il docente chiede loro di indovinare. Gli studenti nominano le canzoni di Laura Pausini, Eros Ramazzotti, Tiziano Ferro, Andrea Bocelli, ecc., ma nessuno sa dirle in italiano. Il docente chiede se conoscono Fabrizio De André; non avendo, purtroppo, nessuna risposta, decide di condivide subito con loro l'UdA dedicata alla canzone Ballata dell'amore cieco e si inizia con il leggere una breve biografia del cantautore.

Durante: si svolgono le attività proposte: (1) attività di accoppiamento immagini e frasi relative a "che cosa fare per amore" e di discussione in gruppi: "che cosa faresti per amore?" (2). Dopo la fase di riscaldamento si passa alla fase di globalità facendo ascoltare la canzone. Nessuno conosceva le canzoni di De André, ma il brano è subito piaciuto, generando commenti positivi da parte degli studenti: la motivazione è alta.

Si svolgono le attività di comprensione vero/falso (3) e di completamento (4). Successivamente agli studenti viene consegnato il testo della canzone e si passa alla fase di analisi: si chiede loro di scrivere la parola corretta (annerita nel testo della canzone) e la sua definizione (5). Al termine di questa attività si procede all'analisi grammaticale costituita da attività di ripasso: trovare nel testo i verbi al passato remoto (6), completare la tabella di coniugazione dei verbi regolari (7) e coniugare i verbi tra parentesi al passato remoto (8). Si svolgono tutte le attività senza nessun tipo di problema, gli studenti partecipano attivamente e sono sempre molto motivati. 
Dopo: una volta terminate le attività di analisi lessicale e grammaticali, si passa alle attività di riflessione/sintesi: si chiede agli studenti di interpretare che cosa volesse dire il cantautore con l'espressione "Voleva un'altra prova del suo cieco amore" (9); quest'attività si svolge in piccoli gruppi e allo scadere del tempo assegnato ogni gruppo espone le sue conclusioni in plenum. Quest'attività ha come l'obiettivo promuovere la discussione rispettando le opinioni dei compagni e riflettendo sulla propria esperienza.

Si passa poi a un'attività di riscrittura creativa in cui si chiede agli studenti di modificare il testo della canzone trasformandola in una Ballata dell'amore vero (10). Qui entra in gioco la riflessione sulla propria esperienza e sui valori che si ritiene legati all'amore vero.

Infine il docente chiede agli studenti che cosa pensano della canzone; essi rispondono che non conoscevano il cantautore, ma che la canzone è divertente e che, allo stesso tempo, induce a riflettere. Le studentesse sono le prime a prendere la parola; per loro l'amore vero è diverso: "lui non è innamorato, è ossessionato", "lei ha qualcosa nella testa, nel cuore...forse ha sofferto da giovane", "per amore non si può uccidere; amare vuole dire vita, vivere". Sollecitato ad intervenire, uno studente afferma che "Lui e lei sono pazzi". Alla richiesta di motivare il suo giudizio, lo studente risponde che è un po' difficile per lui dare una spiegazione in italiano, ma con l'aiuto e suggerimenti dell'insegnante, attraverso la chat privata di Zoom, chiarisce che cosa volesse intendere con la sua affermazione: "la canzone racconta la storia di un nomo onesto che per amore uccide la mamma, probabilmente era un giovane, con poca esperienza e forse anche aveva l'autostima bassa". Immediatamente gli altri studenti iniziano ad inviare le proprie opinioni. Si riportano alcune tra quelle più significative e che rinchiudono i pensieri della classe: "a volte quando non abbiamo autostima alta possiamo fare quello che vogliono altr", "è importante avere amici che ci possano dare dei consigli", "in Perù a volte i giovani si innamorano e hanno anche figll", "non esiste neanche educazione sessuale e aiuto psicologico".

La canzone di André è piaciuta molto e, per questo, gli studenti cercheranno su YouTube altre canzoni del cantautore genovese. Al termine dell'UdA, l'insegnante assegna agli studenti un'attività da svolgere a casa (un cruciverba) per ripassare il passato remoto.

\subsubsection{I cento passi dei Modena City Ramblers}

La canzone I cento passi 5 fa parte della colonna sonora dell'omonimo film che racconta la vita e l'omicidio di Peppino Impastato, giovane attivista impegnato nella lotta contro la mafia nella sua terra, la Sicilia. Una storia tratta dunque dalla vita reale. Il materiale è stato pensato per il livello B2 del QCER e l'UdA ha come obiettivo "socioculturale" quello di riflettere su quanto accaduto in Italia e in Perù nella lotta contro l'ingiustizia e la mafia. Dal punto linguistico invece ha come obiettivo quello di arricchire il lessico e di ripassare il passato prossimo e l'imperfetto indicativo dei verbi.

Prima: l'insegnante chiede agli studenti se hanno partecipato alle ultime manifestazioni svolte in Perù (in riferimento alle proteste del novembre del 2020) ${ }^{6}$, alcuni di loro rispondono di sì e altri di no. Per la fase di riscaldamento, il docente chiede cosa pensano di tutto quello che è accaduto negli ultimi giorni e, dopo aver sentito il loro parere, inizia proiettando delle immagini attraverso Meet ("il funerale di Peppino Impastato", "Peppino alla radio", "Peppino protestando" e "Peppino e suo padre") e chiede di indicare che cosa osservano (1).

5 https://www.youtube.com/watch?v=wE6a-cT6oss.

${ }^{6}$ https://es.wikipedia.org/wiki/Protestas_en_Per\%OC3\%BA_de_2020. 
Durante: per la fase della globalità l'insegnante fa ascoltare la canzone e chiede di segnare le parole ascoltate (2). Nella successiva fase di analisi, fa ascoltare ancora una volta la canzone e chiede di completare una delle strofe; dopo la verifica fa lavorare in piccoli gruppi per cercare di interpretarne il significato (3). Gli studenti lavorano in coppia o in piccoli gruppi e successivamente in plenum usando Meet. Nell'attività successiva gli studenti devono ascoltare ancora una volta la canzone e completare gli spazi vuoti del testo (4). Seguono due attività: la prima per trascrivere in una tabella i verbi al passato prossimo e all'imperfetto indicativo (5) e la seconda per discutere in piccoli gruppi quando usare i due tempi verbali e proporre degli esempi (6). Subito dopo si passa all'attività di analisi lessicale dove gli studenti devono scrivere il sinonimo (annerito nel testo della canzone) accanto a una lista di parole (7).

Dopo: l'insegnate chiede di lavorare in piccoli gruppi e di commentare una parte del testo della canzone "Era la notte buia dello Stato italiano, quella del nove maggio settantotto.../La notte di via Caetani, del corpo di Aldo Moro, l'alba dei funerali di uno stato..." e di rispondere alla domanda: "Anche nella tua nazione c'è stata una notte buia?" (8). L'importante qui è incentivare la discussione in piccoli gruppi e far parlare gli studenti in libertà. Gli studenti sono altamente motivati; dopo il lavoro in gruppo fanno riferimento alle proteste del novembre 2020; a mo' di esempio si riportano qui alcuni dei loro interventi: "professore, una notte buia è la marcia (manifestazione) contro Merino", "è un dittatore, sono morti due giovani", "professore, però molti non partecipano, non aiutano", "alcuni professori non banno dato il permesso per andare alla protesta", "dobbiamo continuare, perché ci sono molti problemi", "ci sono molti giovani che non vogliono partecipare, hanno paura".

Dopo aver riportato le riflessioni dei singoli gruppi in plenum, si passa all'ultima attività: si chiede di spiegare per iscritto cosa narra la canzone (9); scaduto il tempo assegnato per svolgere il lavoro, ogni gruppo legge il testo che ha scritto e, per concludere, il docente riporta sulla lavagna virtuale le interpretazioni comuni a tutti i gruppi e quelle che risultano diverse, per un commento finale.

Gli studenti sono altamente motivati, sin dall'inizio apprezzano la canzone e subito collegano ciò che vi si narra alla loro realtà facendo paragoni con le proteste e i fatti accaduti in Perù: la morte di due giovani studenti e che cosa bisogna fare per cambiare la realtà peruviana. Si capisce che usano il traduttore perché iniziano a usare un lessico ricercato (piuttosto che, consapevolezza, dimettersi, rimuovere, scontro, ecc.) e anche strutture grammaticali di livello medio alto/avanzato (forma passiva, infinito passato, gerundio passato, passato remoto; periodo ipotetico, ecc.); lo fanno con l'obiettivo di comunicare nel modo migliore ciò che pensano. L'attività 10: formulare ipotesi sul titolo della canzone, viene assegnata come compito a casa e servirà per introdurre la nuova UdA utilizzando il film I cento passi.

Alla fine della lezione il docente chiede agli studenti che cosa pensano della canzone. Gli studenti rispondono che è molto interessante, bella e che aiuta a riflettere sulla realtà peruviana; ci sono commenti come: "professore, non sapevo che anche in Italia esistono proteste", "...tutti vogliamo vivere in una società migliore per questo le proteste". Chiedono inoltre se anche nella società odierna italiana vi sono manifestazioni di lotta contro l'ingiustizia, contro la mafia. Uno studente chiede se I cento passi è una canzone di protesta e se è come "Bella ciao". 


\subsubsection{Che fantastica storia è la vita di Antonello Venditti}

Questa canzone racconta in breve la vita di quattro persone e le cose fantastiche che hanno nella loro vita. L'UdA elaborata è pensata per il livello A2 e ha come obiettivo quello di incentivare gli studenti a riflettere sulla loro esperienza di vita. L'obiettivo linguistico-comunicativo è l'arricchimento del repertorio lessicale e lo sviluppo dell'abilità di interazione verbale in italiano con i compagni.

Prima: l'insegnante, dopo aver dato il benvenuto agli studenti non appena si collegano a Meet, consegna l'UdA e fa leggere una breve biografia di Antonello Venditti. Subito dopo si passa alla fase di riscaldamento che consiste nel risolvere degli anagrammi sui nomi delle professioni e mestieri (1). Dopo fa ricordare come si chiede quale professione svolge una persona (2), attività che servirà a far interagire gli studenti chiedendo il nome, come stanno, età, professione e provenienza dei loro compagni; per fare questo si usa una chat interattiva: Spatial chat (3).

Durante: per la fase della globalità si fa ascoltare la canzone e si chiede di identificare i personaggi nominati con i rispettivi dati personali (4); segue la fase di controllo per passare poi alla fase di analisi dove gli studenti ascoltano ancora una volta la canzone e completano gli spazi vuoti del testo (5). Terminata questa attività, si chiede di leggere ancora una volta il testo per identificare il sinonimo di alcune parole annerite nel testo della canzone (6). Per una comprensione più approfondita del messaggio della canzone si chiede agli studenti di leggere due strofe della canzone e di spiegare che cosa intende comunicare il cantautore (7). Per il controllo si chiede agli studenti di confrontarsi in piccoli gruppi e, successivamente, in plenum. La partecipazione è sempre attiva e gli studenti cercano di usare la lingua italiana per comunicare le loro idee, si offrono volontari per poter intervenire. La motivazione è alta.

Dopo: gli studenti lavorano in coppia: il primo svolge il ruolo di intervistatore, il secondo quello di uno dei personaggi della canzone (8). Gli studenti hanno dieci minuti per preparare l'intervista per poi recitarla in plenum. Per finire, si chiede a ogni partecipante di scrivere un breve testo nel quale devono indicare perché per loro è fantastica la vita (9).

Prima di porre termine all'incontro l'insegnante presenta un album virtuale dove ha postato alcune foto della classe (che previamente si è fatto procurare dalla delegata del corso). L'insegnante invita gli studenti a postare una loro foto e a scrivere una presentazione di sé come nella canzone. L'insegnante chiude la riunione video e nota che molti studenti iniziano subito a postare le loro foto e a scrivere il loro profilo.

\subsubsection{Pensa di Francesco Moro}

È una canzone che invita a riflettere sulla mafia e su ogni forma di corruzione e di oppressione e che, ricordando chi non si è arreso all'ingiustizia, invia un messaggio di impegno civile ai giovani. L'UdA elaborata è pensata per il livello B1 e ha come obiettivo sviluppare negli studenti la autoconsapevolezza di quello che possono fare per aiutare ad avere una società migliore. Questa UdA, oltre ad avere l'obiettivo linguistico di arricchire il lessico, mira soprattutto allo sviluppo del pensiero critico.

Prima: prima di iniziare, l'insegnante fa una breve riflessione sulla morte degli studenti durante le manifestazioni che si sono svolte in Perù nel 2020. Nessuno parla fino a quando l'insegnante chiede agli studenti di esprimere il loro parere, ma soltanto due studenti intervengono molto brevemente. L'insegnante presenta la prima attività che consiste 
nell'osservare delle immagini (una statua, la guerra, un cuore, un angelo, una donna con gli occhi coperti e un ragazzo che, in una manifestazione, tiene in mano la bandiera del Perù) e nell'esprimere a che cosa fanno pensare (1). Segue un esercizio in cui si presenta una serie di parole in disordine e si chiede agli studenti di scrivere le frasi in modo corretto (2); per la verifica l'insegnante fa ascoltare la canzone, dando inizio in questo modo alla fase della globalità.

Durante: L'insegnante fa ascoltare nuovamente la canzone e chiede di completare gli spazi vuoti (3). Segue un esercizio che chiede, dopo aver riletto il testo, di selezionare le parole a seconda che siano negative o positive (4). L'obiettivo è quello di far rileggere il testo e di invitare gli studenti a dare un significato (anche personale) alle parole e, in questo modo, di incentivare il pensiero critico. Segue un'attività di analisi del lessico: gli studenti leggono delle definizioni a cui associare la parola corretta, annerita nel testo della canzone (5). Per una comprensione più approfondita si chiede agli studenti di spiegare una strofa della canzone (6) e di rispondere a delle domande aperte (7). L'obiettivo è che gli studenti possano non solo rileggere analiticamente il testo ma anche esprimere le loro opinioni; l'attività viene prima svolta in piccoli gruppi e successivamente in plenum, in modo che vi siano due momenti di confronto. Tutti gli apprendenti vogliono esprimere le loro opinioni. Una studentessa esclama: "forte la canzone". Le diverse attività di comprensione e di analisi hanno fatto in modo che gli studenti si siano identificati con il testo della canzone; molti manifestano il bisogno di lottare contro l'ingiustizia, riportano e commentano la morte di due ragazzi avvenuta durante le manifestazioni del novembre 2020, e, inoltre sono d'accordo nel ritenere importante che anche nella vita privata ognuno debba sapere pensare e riflettere prima di prendere una qualsiasi decisione.

Dopo: per finire, l'insegnante chiede agli studenti di elaborare un'intervista: uno studente svolge il ruolo di giornalista e l'altro quello di un personaggio che ha lasciato un segno nella storia dell'umanità (8). Dopo 15 minuti di preparazione, ogni coppia mette in scena l'intervista; quasi tutti gli studenti hanno scelto personaggi peruviani, due coppie hanno scelto Gesù e Mahatma Gandhi.

\section{CONCLUSIONI}

Nel processo di insegnamento/apprendimento di una lingua straniera è importante mantenere alta la motivazione e a questo scopo è possibile utilizzare la canzone perché, è un testo autentico ricco di elementi linguistici e culturali e perché piace a tutti, ma nel momento della sua didattizzazione bisogna considerare i destinatari e i loro bisogni comunicativi, linguistici e socio-culturali. Ė inoltre importante analizzare le caratteristiche del testo e della musica della canzone facendo attenzione al tipo di lingua (varietà, registro, ecc.) usato dall'autore e ai riferimenti storici, sociali e culturali presenti o impliciti: la presenza di elementi linguistici (lessicali, morfosintattici, testuali) e socio-culturali a volte poco noti.

La sperimentazione delle UdA hanno permesso di rilevare che la canzone può essere utile per affrontare aspetti sociali e riflettere sulla propria realtà e sulla propria esperienza di vita, facendo parlare gli studenti su quello che pensano e vivono. La canzone permette di intervenire su aspetti sociali e di riflettere su quanto accade in altre società e, a confronto, nella propria; per questi motivi si è scelto di far ascoltare delle canzoni italiane in classe, con il fine di sviluppare competenze non solo linguistico-comunicative ma anche socio-culturali. 
(C) Italiano LinguaDue 2. 2021. H. Tolentino Quiñones, Uso della canzone nella classe di lingua e cultura per sviluppare la competenza socioculturale

\section{RIFERIMENTI BIBLIOGRAFICI}

Allomello C. (2015), Il sostegno della musica nei processi di apprendimento, APIM, Torino.

Balboni P. E. (2002), Le sfide di Babele, UTET, Torino.

Balboni P. E. (2018), "Sillabo di riferimento per l'insegnamento dell'italiano della musica", in Studi sull'apprendimento e l'insegnamento linguistico (SAIL, 14), Edizioni Ca' Foscari, Venezia.

Begotti P. (n.d.), Didattizzazione di materiali autentici e analisi dei manuali di italiano per stranieri, Università ca' Foscari, Venezia.

Beozzo J. (2013), L’influenza della musica nello sviluppo cognitivo e linguistico del bambino, Università degli studi di Verona, Verona.

Berrettini L. (2009), Modelli operativi per la didattica: l'organizzazione di unità di apprendimento, Icon, Pisa.

Caon F. (n.d.), Canzone pop e canzone d'autore per la didattica della lingua, della cultura italiana e per l'approccio allo studio della letteratura, Università Ca' Foscari - FILIM, Venezia.

Consejo de Europa (2002), Marco Común Europeo De Referencia Para Las Lenguas, Centro Virtual Cervantes, Madrid.

Coonan C. (n.d.), La ricerca-az̧one, Università Ca' Foscari, Venezia: https://www.academia.edu/3311353/La_ricerca_azione.

Custodio N., Cano-Campos, M. (2017), "Efectos de la música sobre las funciones cognitivas", in Reuropsoquiart, 80, 1, pp. 60-70.

Mamani Quispe E. Z. (2018), Habilidades de canto y expresión oral en idioma inglés en los estudiantes de la E.P de arquitectura y urbanismo de la UNA - Puno 2018, Universidad Nacional del Altiplano, Puno.

Mauroni E. (2011), "Imparare l'italiano L2 con le canzoni. Un contributo didattico", in Italiano LinguaDue, 2, pp. 395-438:

https://riviste.unimi.it/index.php/promoitals/article/view/1242.

Ministero degli Affari Esteri e della Cooperazione Internazionale (2018), L'italiano nel mondo che cambia - 2018, Roma:

https://www.esteri.it/mae/resource/doc/2018/10/rapporto_2018_li.pdf.

Novello A. (n.d.), "Modelli operativi per l'insegnamento dell'italiano lingua straniera", in FILIM - Formazione degli Insegnanti di Lingua Italiana nel Mondo, Ca’ Foscari, Venezia.

Oliveira H. F., Silva, J. C. V. (2018), "Letramento musical de profesores de lingua estrangeira (ingles)", in Educação Em Revista, 34, e182650:

https://www.scielo.br/j/edur/a/y4FDjGHt7JxgQvz7PdGScqt/?lang=pt\&forma $\mathrm{t}=$ pdf.

Pichiassi M (1999), Fondamenti di Glottodidattica, Guerra-Soleil, Perugia.

Sánchez L. M., Díaz, Chasterlain J. A. (2019), "La música, el oído y el habla”, in Revista electrónica científico-pedagógica Ciencias Pedagògicas, 3, 4, pp. 99-108.

Torresan P. (n.d.), "Tecnologie per l'apprendimento dell'italiano", in FILIM - Formazione degli Insegnanti di Lingua Italiana nel Mondo, Ca' Foscari, Venezia.

Vidal M., Rivera N. (2007), "Investigación-acción”, Educación Médica Superior, 21,4 (s.p.): http:/ / scielo.sld.cu/scielo.php?script=sci_arttext\&pid=S0864-

21412007000400012. 


\section{APPENDICE: I MATERIALI E LE ATTIVITÀ}

\section{LA BALLATA DELL'AMORE CIECO}

\section{Fabrizio De André}

https://www.youtube.com/watch?v=HXE8waG_X-4.

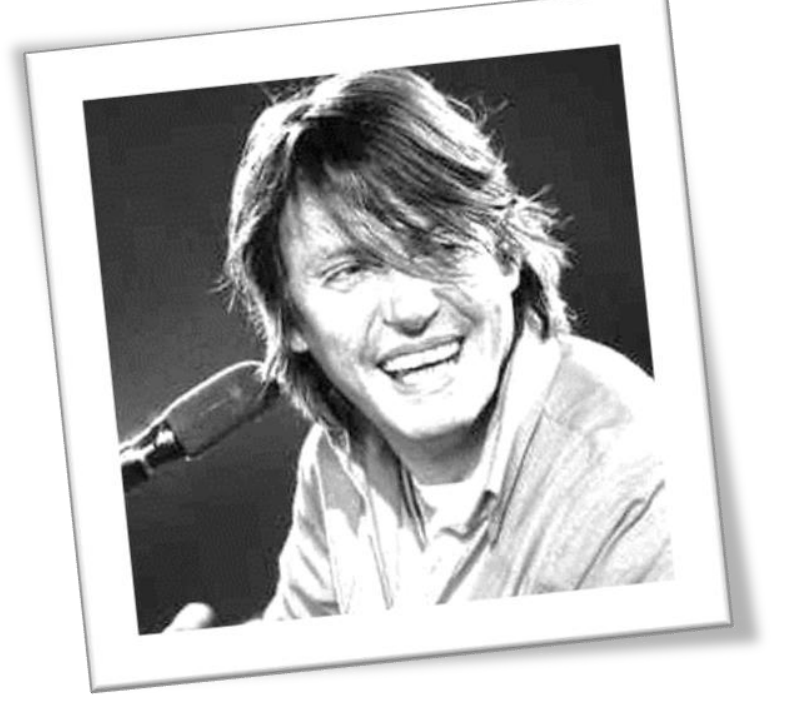

Fabrizio Cristiano De André, noto come Fabrizio De André (Genova, 18 febbraio 1940 - Milano, 11 gennaio 1999), è stato un cantautore italiano.

Considerato da molti critici musicali uno dei più importanti e influenti cantautori italiani.

De André ha inciso quattordici album in studio, più alcune canzoni pubblicate solo come singoli e poi riedite in antologie. Molti testi delle sue canzoni raccontano storie di emarginati, ribelli e prostitute, e sono considerate da alcuni critici vere e proprie poesie, tanto da essere inserite in varie antologie scolastiche di letteratura.

La rivista Rolling Stone Italia lo posiziona al secondo posto tra i migliori artisti musicali italiani di sempre. Insieme a Bruno Lauzi, Gino Paoli, Umberto Bindi e Luigi Tenco è uno degli esponenti della cosiddetta Scuola genovese, un nucleo di artisti che rinnovò profondamente la musica leggera italiana

Di idee anarchiche e pacifiste, è stato anche uno degli artisti che maggiormente hanno valorizzato la lingua ligure. Ha affrontato inoltre, in misura minore e differente, altri idiomi, come il gallurese e il napoletano. Durante la sua carriera ha collaborato con molte personalità della cultura e importanti artisti della scena musicale e culturale italiana. 
(C) Italiano LinguaDue 2. 2021. H. Tolentino Quiñones, Uso della can₹one nella classe di lingua e cultura per sviluppare la competenza socioculturale

\section{La ballata dell'amore cieco}

Un uomo onesto, un uomo probo

Tralalalalla tralallaleru

S'innamorò perdutamente

D'una che non lo amava niente

Gli disse: "Portami domani"

Tralalalalla tralallaleru

Gli disse: "Portami domani"

"Il cuore di tua madre per i miei cani"

Lui dalla madre andò e l'uccise

Tralalalalla tralallaleru

Dal petto il cuore le strappò

$\mathrm{E}$ dal suo amore ritornò

Non era il cuore, non era il cuore

Tralalalalla tralallaleru

Non le bastava quell'orrore

Voleva un'altra prova del suo cieco amore

Gli disse: "Amor, se mi vuoi bene"

Tralalalalla tralallaleru

Gli disse: "Amor, se mi vuoi bene"

"Tagliati dai polsi le quattro vene"

Le vene ai polsi lui si tagliò

Tralalalalla tralallaleru

E come il sangue ne sgorgò

Correndo come un pazzo da lei tornò

Gli disse lei, ridendo forte

Tralalalalla tralallaleru

Gli disse lei, ridendo forte

"L'ultima tua prova sarà la morte"

E mentre il sangue lento usciva

E ormai cambiava il suo colore

La vanità fredda gioiva

Un uomo s'era ucciso per il suo amore

Fuori soffiava dolce il vento

Tralalalalla tralallaleru

Ma lei fu presa da sgomento

Quando lo vide morir contento

Morir contento e innamorato

Quando a lei niente era restato

Non il suo amore, non il suo bene

Ma solo il sangue secco delle sue vene 
(C) Italiano LinguaDue 2. 2021. H. Tolentino Quiñones, Uso della can₹one nella classe di lingua e cultura per sviluppare la competenza socioculturale

1. Cosa fare per amore? Unisci le frasi alle immagini.
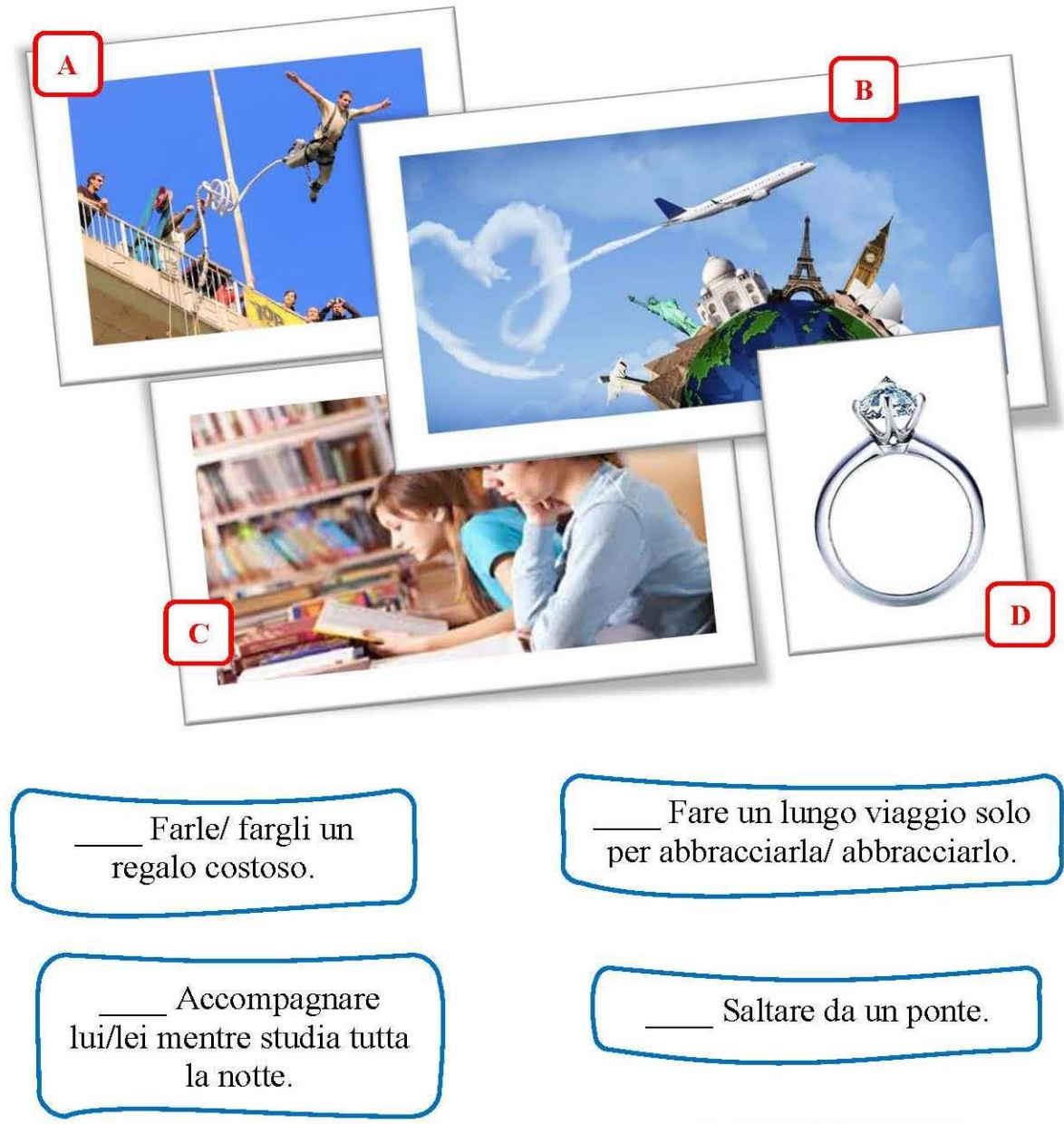

Fare un lungo viaggio solo per abbracciarla/ abbracciarlo.

\section{Cosa faresti per amore?}

Lavora in piccoli gruppi,

e rispondi alla domanda.

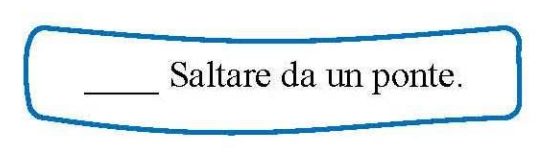

3. Ascolta la canzone e rispondi con vero (V) o falso (F).
a) Un uomo si innamorò di una donna che non lo amava.
$\begin{array}{ll}\mathrm{V} & \mathrm{F} \\ \mathrm{V} & \mathrm{F}\end{array}$
b) La donna era una principessa.
$\mathrm{V} \quad \mathrm{F}$
c) La donna chiese molte prove d'amore.
d) Una delle prove era uccidere dei cani.
e) Alla fine, tutti e due vissero felici per sempre.

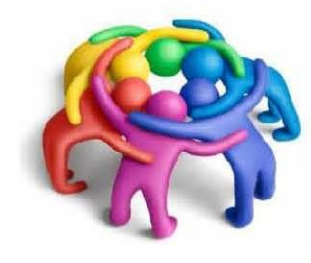


(C) Italiano LinguaDue 2. 2021. H. Tolentino Quiñones, Uso della canzone nella classe di lingua e cultura per sviluppare la competenza socioculturale

\section{Ascolta la canzone e completa gli spazi vuoti.}

Un uomo onesto, un uomo probo

Tralalalalla tralallaleru

S'innamorò

D'una che non lo amava niente.

Gli disse portami domani

Tralalalalla tralallaleru

Gli disse portami domani

Il__ per i miei cani.

Lui dalla madre andò e l'uccise

Tralalalalla tralallaleru

Dal petto il cuore le strappò

E dal suo amore ritornò.

Non era il cuore, non era il cuore

Tralalalalla tralallaleru

Non le

Voleva un'altra prova del suo cieco amore.

Gli disse amor se mi vuoi bene

Tralalalalla tralallaleru

Gli disse amor se mi vuoi bene

Tagliati dei polsi le
Le vene ai polsi lui si tagliò

Tralalalalla tralallaleru

E come il sangue ne sgorgò

Correndo come un pazzo da lei tornò.

Gli disse lei

Tralalalalla tralallalero

Gli disse lei ridendo forte,

L'ultima tua prova sarà la morte.

E mentre il sangue lento usciva

E ormai

La vanità fredda gioiva,

Un uomo s'era ucciso per il suo amore.

Fuori soffiava dolce il vento

Tralalalalla tralallaleru

Ma lei fu presa da sgomento

Quando lo vide morir contento.

Morir contento e innamorato

Quando a lei

Non il suo amore non il suo bene

Ma solo il sangue secco delle sue vene.

5. Leggi il testo della canzone e trascrivi le parole/espressioni in grassetto accanto alla definizione corretta.

\begin{tabular}{|l|l|}
\hline \multicolumn{1}{|c|}{ Parola/espressione } & \multicolumn{1}{|c|}{ Definizione } \\
\hline & La zona sopra la mano, dove si vede l'arteria. \\
\hline & Che ha perso la ragione; malato di mente. \\
\hline & Turbamento, confusione. \\
\hline & Che vive e agisce onestamente. \\
\hline & Provare piacere, allegria. \\
\hline & Togliere bruscamente qualcosa a qualcuno. \\
\hline & $\begin{array}{l}\text { Nel desiderio di essere ammirato per le proprie } \\
\text { qualità, spesso solo presunte. }\end{array}$ \\
\hline & Detto di liquidi, uscire, scaturire in abbondanza. \\
\hline
\end{tabular}


(C) Italiano LinguaDue 2. 2021. H. Tolentino Quiñones, Uso della can₹one nella classe di lingua e cultura per sviluppare la competenza socioculturale

6. Ricordi il passato remoto? Leggi nuovamente il testo della canzone e trova tutti i verbi al passato remoto. Dopo, completa la tabella come nell'esempio.

\begin{tabular}{|l|l|l|l|}
\hline Passato remoto & Verbo all'infinito & Passato remoto & Verbo all'infinito \\
\hline Si innamorò & Innamorarsi & & \\
\hline & & & \\
\hline & & & \\
\hline & & & \\
\hline & & & \\
\hline
\end{tabular}

7. Completa la tabella.

\begin{tabular}{|l|l|l|l|}
\cline { 2 - 4 } \multicolumn{1}{c|}{} & \multicolumn{1}{c|}{ RITORNARE } & \multicolumn{1}{c|}{ VENDERE } & \multicolumn{1}{c|}{ DORMIRE } \\
\hline IO & Ritornai & & Dormii \\
\hline TU & & Vendesti & Dormisti \\
\hline LUI/LEI & & Vendé & \\
\hline NOI & Ritornammo & & \\
\hline VOI & & & Dormiste \\
\hline LORO & Ritornarono & Venderono & \\
\hline
\end{tabular}

8. Coniuga i verbi fra parentesi al passato remoto.

1) Carlo (uscire)

2) Molti italiani (emigrare)

3) La mia famiglia (abitare) al Nord.

4) Giulio Cesare (conquistare)

5) Quando (comprare -io)

6) $\mathrm{Tu}$ (ricevere)

7) Non (credere-io)

8) Un vecchio signore (entrare)

9) Dante Alighieri (morire)

10) Quando (ereditare-voi) sbattendo la porta. in America dopo la grande guerra. a Roma fino al 1990, poi (trasferirsi -noi) la Gallia. questa casa, avevo 23 anni. molte lettere dai tuoi ammiratori? mai a quello che dicevano sul tuo conto. nel bar. nel 1321. questo casale? 
(C) Italiano LinguaDue 2. 2021. H. Tolentino Quiñones, Uso della canzone nella classe di lingua e cultura per sviluppare la competenza socioculturale

9. Nel testo si legge: "Voleva un'altra prova del suo cieco amore". In piccoli gruppi discutete su quali potrebbero essere le conseguenze di un amore cieco, dopo fate sapere le vostre conclusioni in plenum.

10. Lavora con un compagno. Modificate la canzone dove credete sia necessario per renderla una "Ballata dell'amore vero"

\section{ATTIVITÀ PER CASA}

1. Trova i verbi al passato remoto.

Verbi al passato remoto

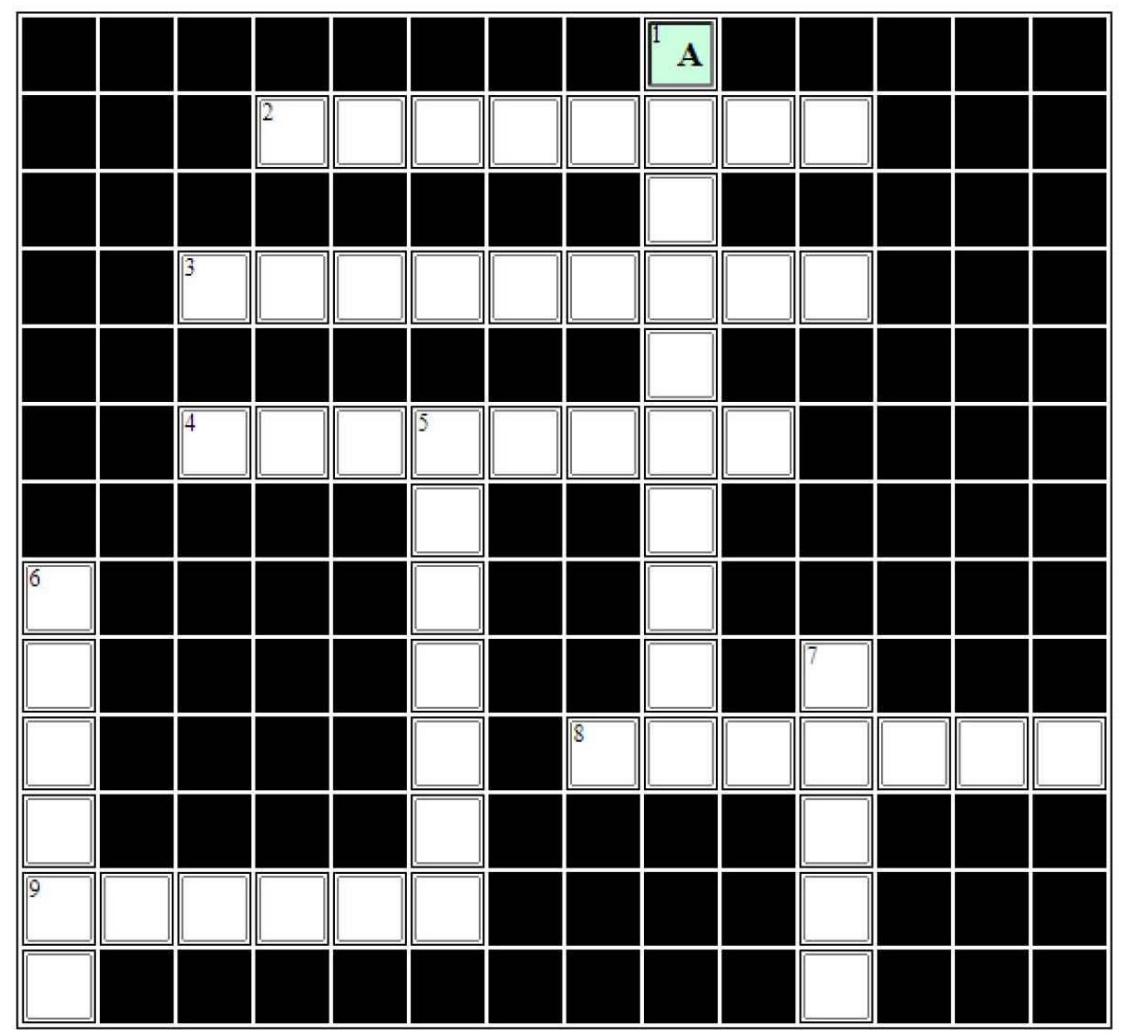

2. SENTIRE - VOI 1. ASCOLTARE - NOI

3. VENDERE - LORO 5. MORIRE - TU

4. DORMIRE - TU 6. CANTARE - IO

8. POTERE - VOI 7. VENDERE - IPJ 
(C) Italiano LinguaDue 2. 2021. H. Tolentino Quiñones, Uso della canzone nella classe di lingua e cultura per sviluppare la competenza socioculturale

\section{CENTO PASSI}

\section{MODENA City RAMBLERS}

https://www.youtube.com/watch?v=wE6a-cT6oss.

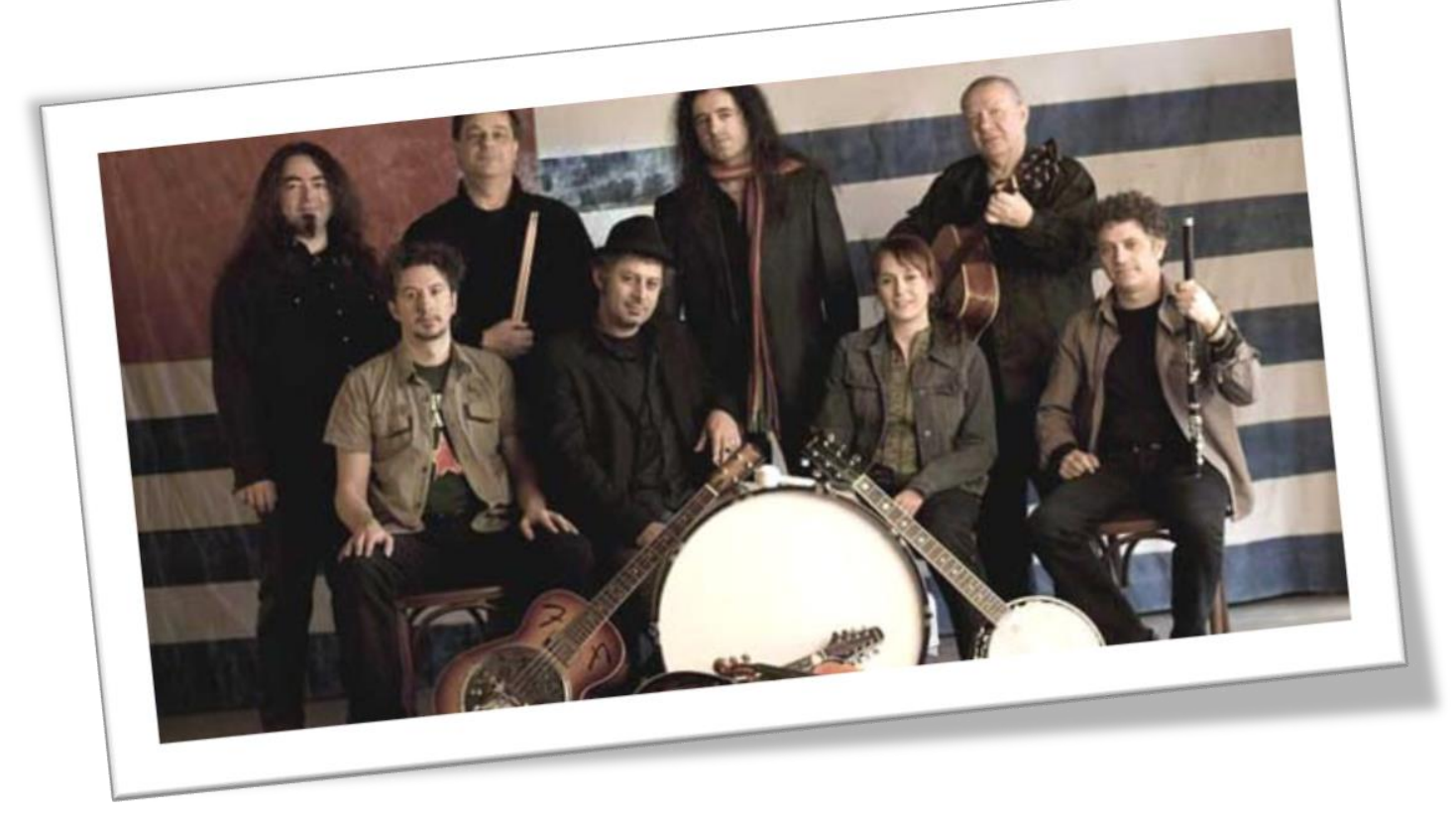

I Modena City Ramblers (anche nella forma abbreviata MCR) sono un gruppo musicale italiano nato nel 1991.

Autodefiniscono il loro genere musicale come combat folk, dichiarando sin dall'esordio un amore incondizionato per il folk irlandese, le cui sonorità rimangono anche dopo l'influenza di altri generi, in particolare il rock con contaminazioni di punk.

Sin dai tempi in cui suonano solo musica irlandese, i Modena City Ramblers, come già i Pogues, utilizzano brani strumentali della tradizione popolare (irlandese, scozzese, celtica e poi anche klezmer, balcanica, italiana) come basi per loro brani, come riff o come assolo. 
(C) Italiano LinguaDue 2. 2021. H. Tolentino Quiñones, Uso della canzone nella classe di lingua e cultura per sviluppare la competenza socioculturale

\section{I cento passi}

È nato nella terra dei vespri e degli aranci Tra Cinisi e Palermo parlava la sua radio Negli occhi si leggeva la voglia di cambiare La voglia di giustizia che lo portò a lottare.

Aveva un cognome ingombrante e rispettato Di certo in quell'ambiente da lui poco onorato Si sa come si nasce ma non come si muore E non se un ideale ti porterà dolore

Ma la tua vita ora puoi cambiare

Solo se sei disposto a camminare

Gridando forte senza aver paura

Contando cento passi lungo la tua strada

E allora

Uno, due, tre, quattro, cinque, dieci, cento passi Uno, due, tre, quattro, cinque, dieci, cento passi Uno, due, tre, quattro, cinque, dieci, cento passi

Poteva come tanti scegliere di partire

Invece lui scelse di restare

Gli amici, la politica, la lotta di partito

Alle elezioni si era candidato

Diceva da vicino non gli avrebbero controllati

Ma poi non ebbe tempo perché venne ammazzato

Il nome di suo padre nella notte non è servito

Gli amici disperati non l'hanno più trovato

E allora dimmi se sai contare

Dimmi se sai anche camminare

Contare camminare insieme a cantare

La storia di Peppino e degli amici siciliani

E allora

Uno, due, tre, quattro, cinque, dieci, cento passi

Uno, due, tre, quattro, cinque, dieci, cento passi

Uno, due, tre, quattro, cinque, dieci, cento passi

Era una notte buia dello stato italiano

Quella del nove maggio Settantotto

La notte di via Caetani e del corpo di Aldo Moro

L'arma dei funerali di uno stato

E allora dimmi se sai contare

Dimmi se sai anche camminare

Contare camminare insieme a cantare

La storia di Peppino e degli amici siciliani

E allora

Uno, due, tre, quattro, cinque, dieci, cento passi

Uno, due, tre, quattro, cinque, dieci, cento passi

Uno, due, tre, quattro, cinque, dieci, cento passi 
(C) Italiano LinguaDue 2. 2021. H. Tolentino Quiñones, Uso della can₹one nella classe di lingua e cultura per sviluppare la competenza socioculturale

1. Osserva le foto e prova a dire cosa osservi. Lavora con un compagno.
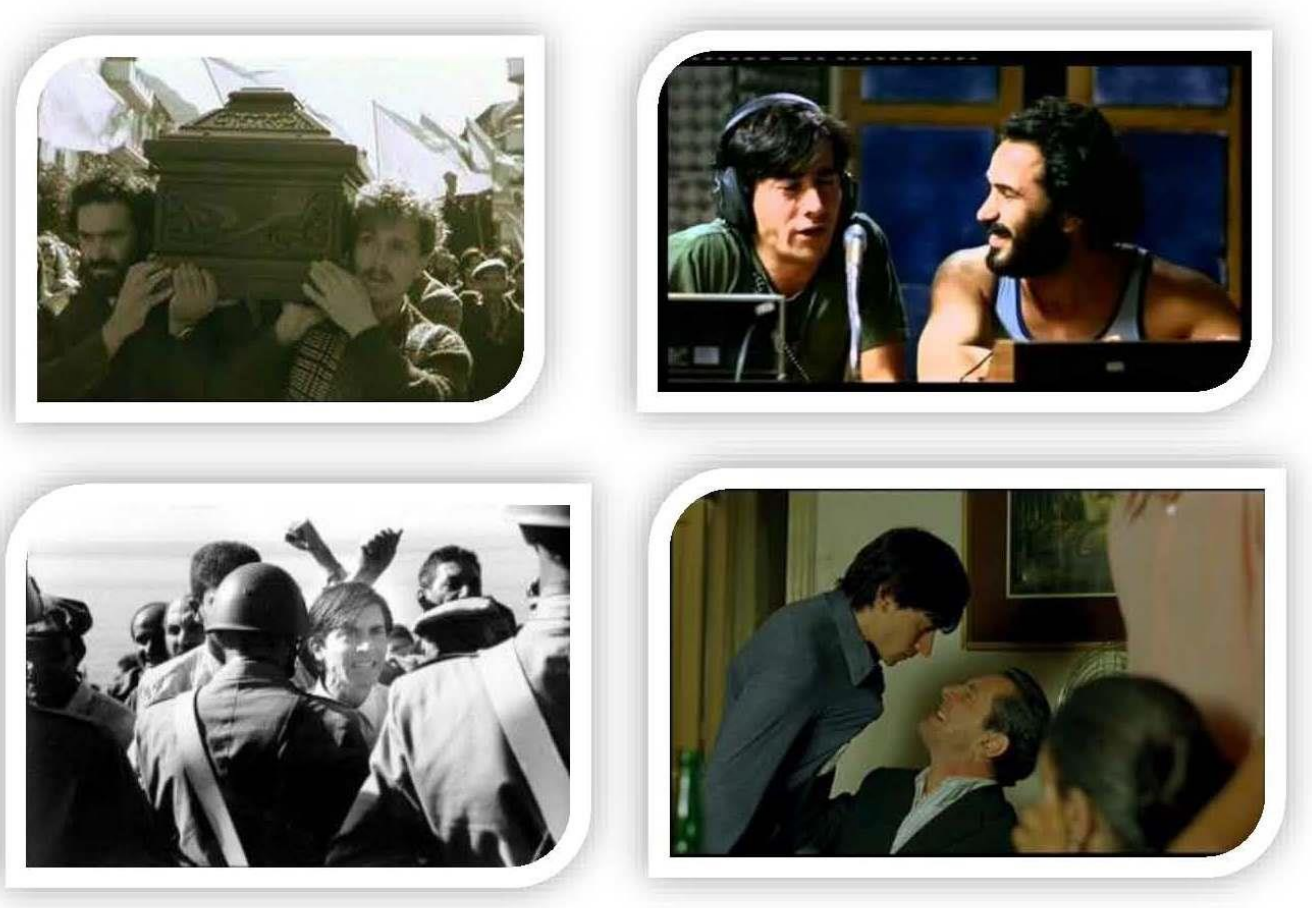

2. Ascolta e segna le parole che senti.

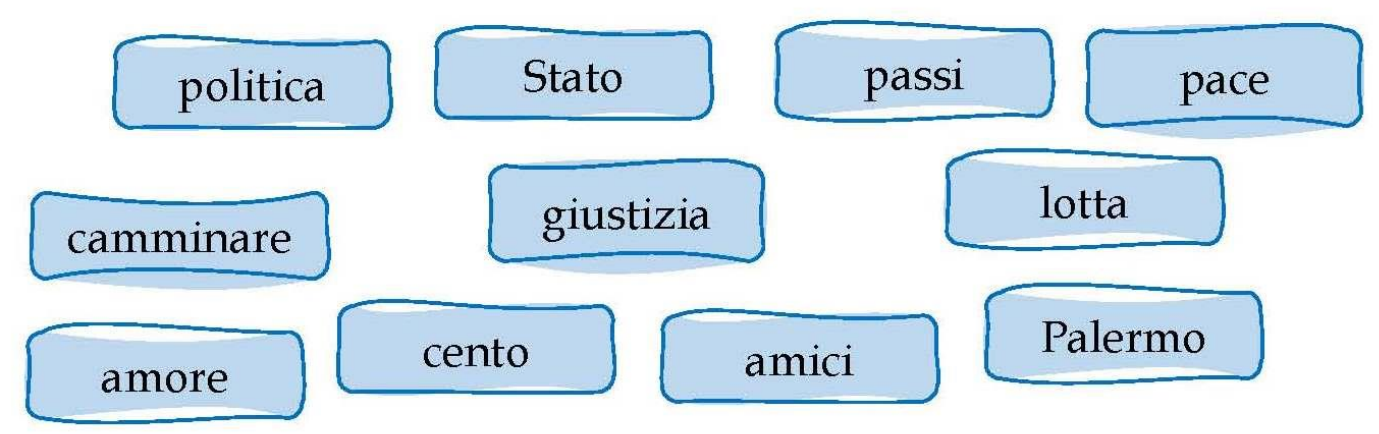

3. Ascolta ancora una volta e completa. Dopo, parla in piccoli gruppi sul significato di questa parte del testo della canzone I cento passi.

\begin{tabular}{l} 
"Ma la tua vita adesso puoi $\_$solo se sei disposto a \\
\hline gridando forte senza aver $\_$contando
\end{tabular}




\section{Ascolta la canzone e completa gli spazi vuoti.}

Nato nella terra dei vespri e degli aranci, tra Cinisi e Palermo alla sua radio...

Negli occhi si la voglia di cambiare, la voglia di Giustizia che lo portò a lottare...

un cognome ingombrante e rispettato, di certo in quell'ambiente da lui poco onorato...

Si sa dove si nasce ma non come si muore e non se un'ideale ti porterà dolore...

"Ma la tua vita adesso puoi cambiare solo se sei disposto a camminare, gridando forte senza aver paura contando cento passi lungo la tua strada" ...

Allora... 1,2,3,4,5,10,100 passi!... 1,2,3,4,5,10,100 passi!

"Noi ci dobbiamo ribellare" (dal film) come tanti scegliere e

partire, invece lui decise di restare... Gli amici, la politica, la lotta del partito... alle elezioni si era candidato... da vicino li avrebbe controllati, ma poi non ebbe tempo perché venne ammazzato...
Il nome di suo padre nella notte non gli amici disperati non $1^{\prime}$ più

"Allora dimmi se tu sai contare, dimmi se sai anche camminare, contare, camminare insieme a cantare la storia di Peppino e degli amici siciliani"...

Allora... 1,2,3,4,5,10,100 passi!... $1,2,3,4,5,10,100$ passi! la notte buia dello Stato italiano, quella del nove maggio settantotto...

La notte di via Caetani, del corpo di Aldo Moro, l'alba dei funerali di uno stato...

"Allora dimmi se tu sai contare, dimmi se sai anche camminare, contare, camminare insieme a cantare la storia di Peppino e degli amici siciliani"...

Allora... 1,2,3,4,5,10,100 passi!... $1,2,3,4,5,10,100$ passi! 
(C) Italiano LinguaDue 2. 2021. H. Tolentino Quiñones, Uso della can₹one nella classe di lingua e cultura per sviluppare la competenza socioculturale

5. I verbi che hai usato per completare la canzone sono al passato prossimo e all'imperfetto indicativo. Trascrivili nelle tabelle e scrivi l'infinito.
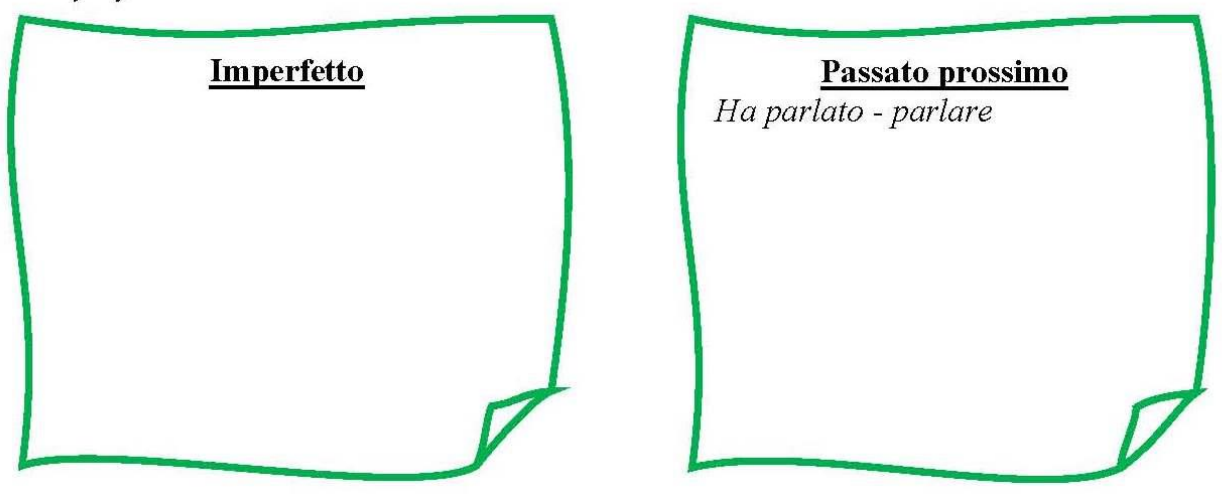

6. Quando si usa il passato prossimo e quando l'imperfetto indicativo? Parlane con un compagno e fate qualche esempio.
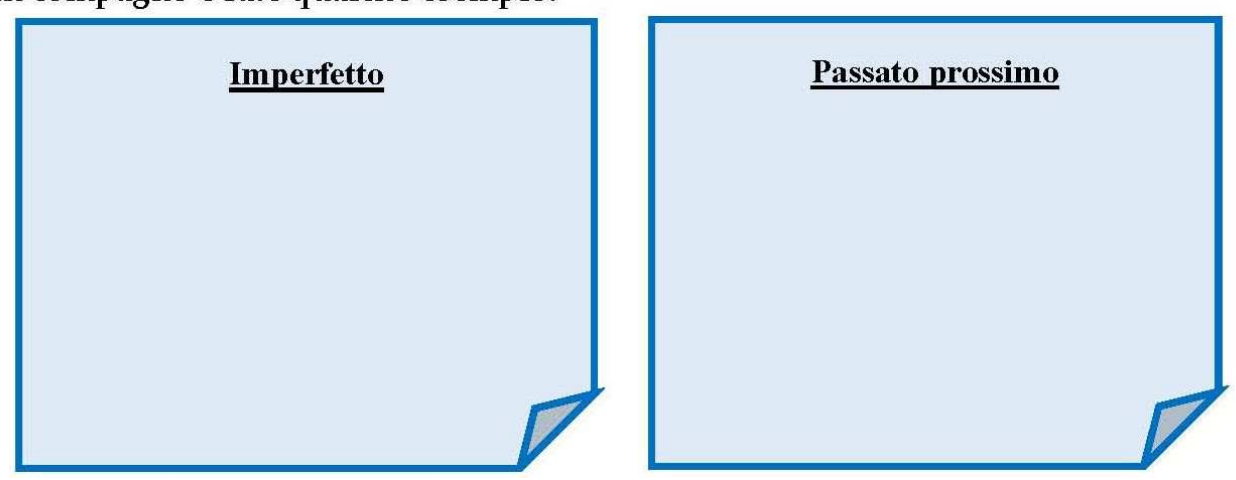

7. Leggi il testo della canzone e scrivi le parole annerite accanto al significato corretto.

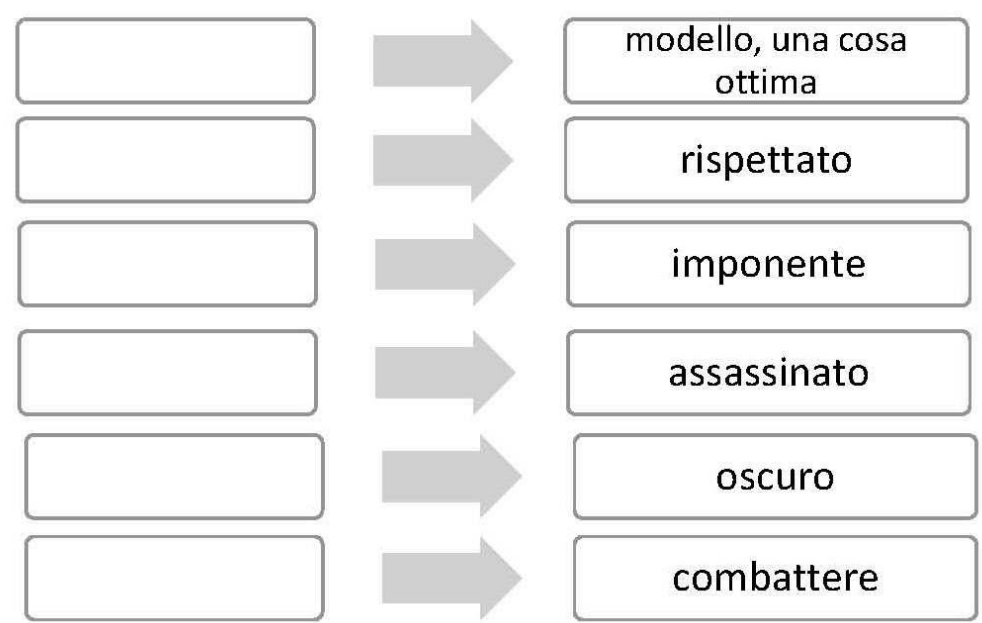


(C) Italiano LinguaDue 2. 2021. H. Tolentino Quiñones, Uso della canzone nella classe di lingua e cultura per sviluppare la competenza socioculturale

8. Leggi questa frase della canzone. Cosa vuol dire? Lavora in piccoli gruppi.

Era la notte buia dello Stato italiano, quella del nove maggio settantotto...

La notte di via Caetani, del corpo di Aldo Moro, l'alba dei funerali di uno stato...

\section{Anche nella tua nazione c'è stata una notta buia?}

9. Cosa narra la canzone? Lavora insieme ad alcuni compagni e spiegate per scritto.

Dopo leggete in plenum le vostre conclusioni. Siete arrivati tutti alla stessa conclusione?

10. In una parte del testo si legge: $1,2,3,4,5,10,100$ passi'... Secondo te, perché?

Prova a fare un'ipotesi. Dopo, fai sapere a tutta la classe le tue ipotesi. 
(C) Italiano LinguaDue 2. 2021. H. Tolentino Quiñones, Uso della canzone nella classe di lingua e cultura per sviluppare la competenza socioculturale

\section{CHE FANTASTICA STORIA È LA VITA ANTONELLO VENDITTI}

https://www.youtube.com/watch?v=fTPtj43aCZU

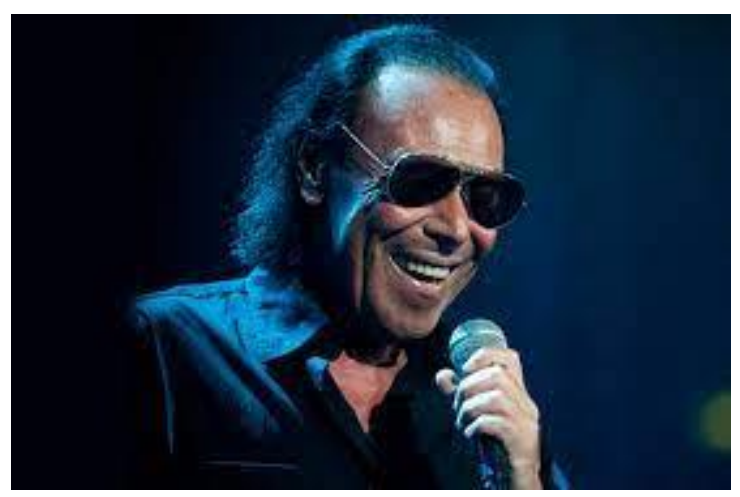

Nato 1'8 marzo 1949 a Roma sotto il segno dei Pesci, ne è diventato il simbolo a livello musicale: re della Capitale, è uno degli artisti della cosiddetta 'Scuola romana'. Oltre 30 milioni di dischi venduti, è tra i più prolifici cantautori della scena italiana, con un debutto e una carriera inarrestabili dal 1972.

Figlio unico di una famiglia medio-borghese, si è avvicinato molto presto alla musica. I genitori sono Vincenzino Italo, ex funzionario di Stato poi diventato prefetto di Roma, e Wanda Sicardi, ex insegnante di greco e latino al liceo. Proprio lei lo ha spronato a intraprendere gli studi di pianoforte.

\section{Che fantastica storia è la vita}

Mi chiamo Antonio e faccio il cantautore

E mio padre e mia madre mi volevano dottore

Ho sfidato il destino per la prima canzone

Ho lasciato gli amici, ho perduto l'amore

E quando penso che sia finita

È proprio allora che comincia la salita

Che fantastica storia è la vita

Mi chiamo Laura e sono laureata

Dopo mille concorsi faccio l'impiegata

E mio padre e mia madre, una sola pensione

Fanno crescere Luca, il mio unico amore

A volte penso che sia finita

Ma è proprio allora che comincia la salita

Che fantastica storia è la vita

Che fantastica storia è la vita

E quando pensi che sia finita

È proprio allora che comincia la salita

Che fantastica storia è la vita

Mi chiamano Gesù e faccio il pescatore

E del mare e del pesce sento ancora l'odore 
(C) Italiano LinguaDue 2. 2021. H. Tolentino Quiñones, Uso della can₹one nella classe di lingua e cultura per sviluppare la competenza socioculturale

Di mio Padre e mia Madre, su questa croce

Nelle notti d'estate, sento ancora la voce

E quando penso che sia finita

È proprio allora che comincia la salita

Che fantastica storia è la vita

Che fantastica storia è la vita

Mi chiamo Aicha, come una canzone

Sono la quarta di tremila persone

Su questo scoglio di buona speranza

Scelgo la vita, l'unica salva

E quando penso che sia finita

È proprio adesso che comincia la salita

Che fantastica storia è la vita

Che fantastica storia è la vita

\section{Ti ricordi le professioni? Risolvi l'anagramma.}

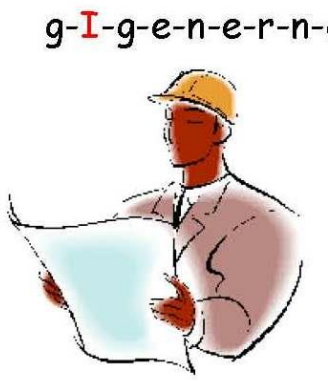

3.

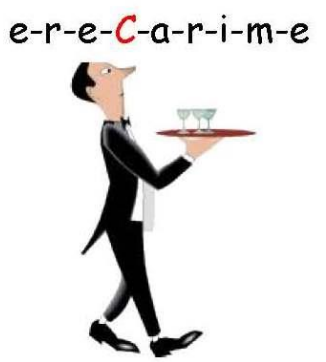

6.

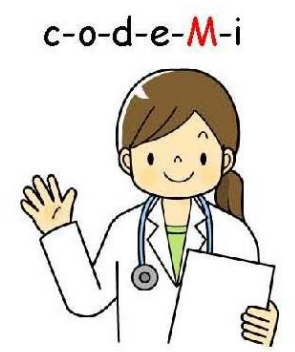

7.

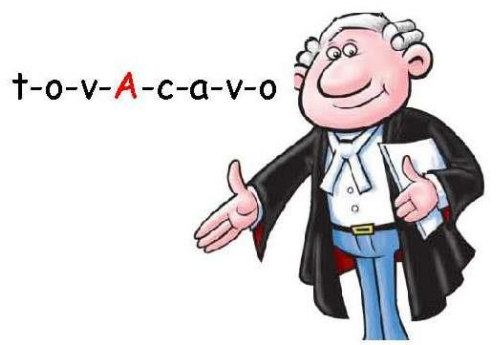

5.

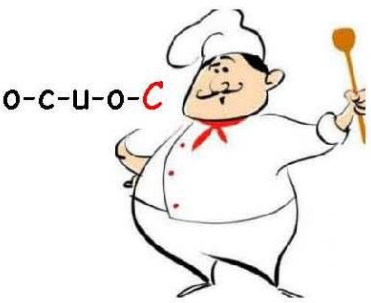

2.

1.

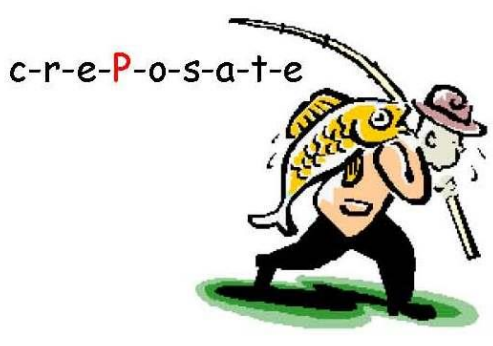

4.

$$
a-\dagger-o-c-a-r-e-c-I-i
$$

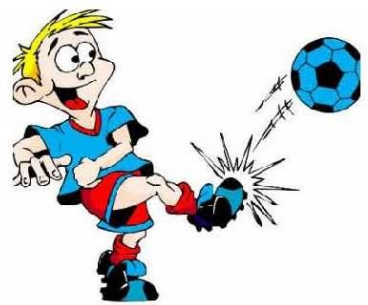

9.

8.
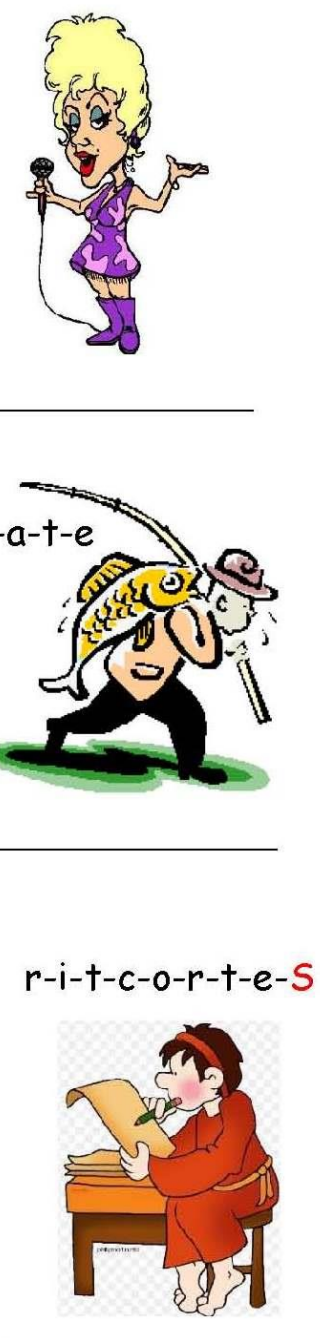

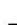


(C) Italiano LinguaDue 2. 2021. H. Tolentino Quiñones, Uso della canzone nella classe di lingua e cultura per sviluppare la competenza socioculturale

2. Come chiedi a una persona il lavoro che fa?

FORMALE: Che _ ?

INFORMALE: Che $?$

Domanda a catena: Chiedi al compagno che lavoro fa.

Come si risponde?

3. Che domande fai a una persona che vuoi conoscere? Completa la tabella e dopo intervista tre compagni.

\begin{tabular}{|l|l|l|l|l|}
\hline \multicolumn{1}{|c|}{ DATI } & DOMANDE & COMPAGNO 1 & COMPAGNO 2 & COMPAGNO 3 \\
\hline Nome & & & & \\
\hline Stato di salute & & & & \\
\hline Età & & & & \\
\hline Professione & & & & \\
\hline Nazione/città & & & & \\
\hline
\end{tabular}

4. Ascolta e scrivi il nome e tutti i dati possibili di ogni persona.

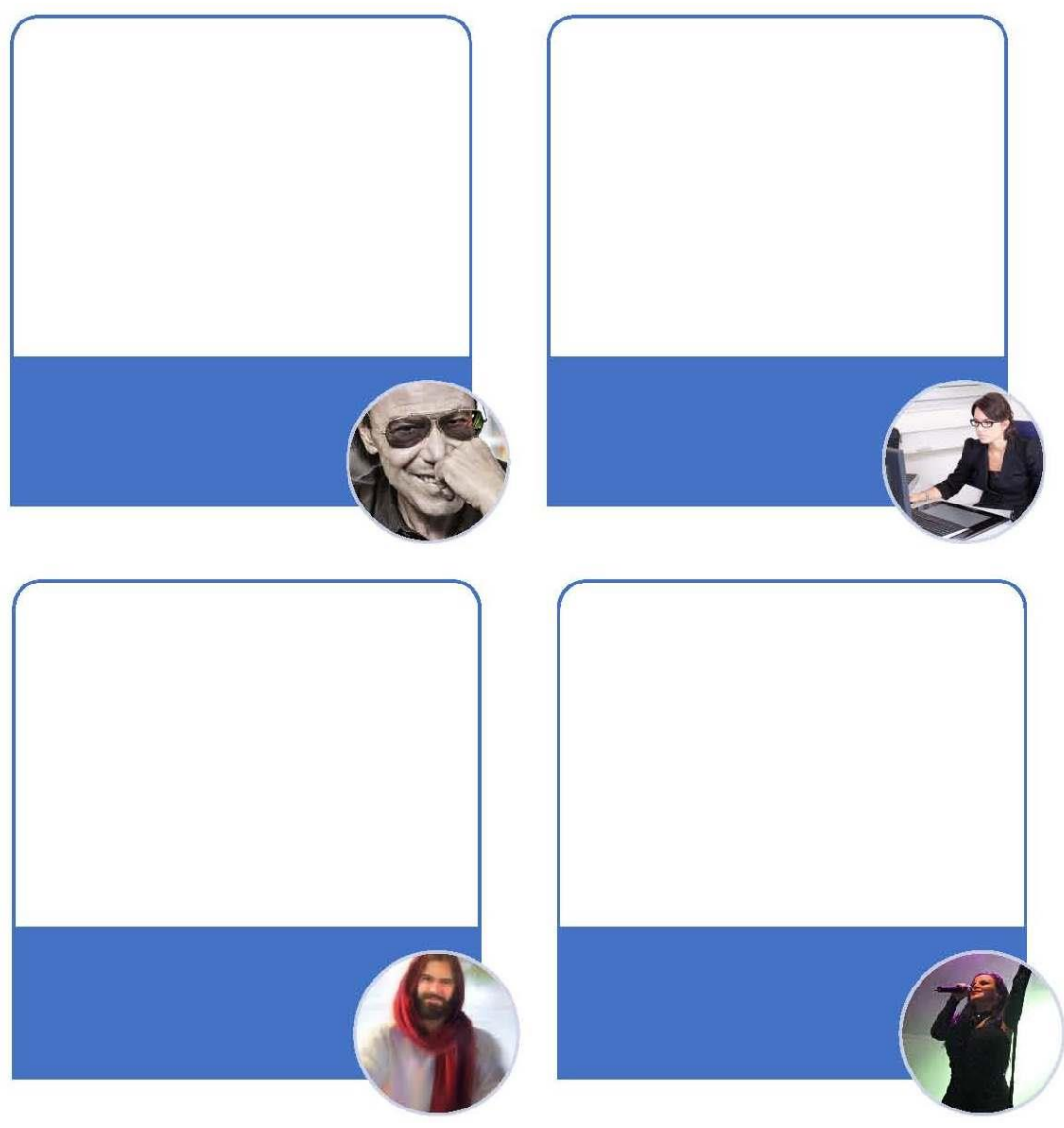


(C) Italiano LinguaDue 2. 2021. H. Tolentino Quiñones, Uso della canzone nella classe di lingua e cultura per sviluppare la competenza socioculturale

\section{Ascolta e completa il testo della canzone.}

\section{Che fantastica storia è la vita \\ Antonello Venditti}

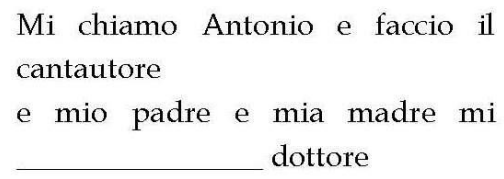

ho sfidato il destino per la prima canzone gli amici,

ho perduto l'amore

E quando penso che sia finita

è proprio allora che comincia la salita

Che fantastica storia è la vita

Mi chiamo Laura e sono

dopo mille concorsi faccio

l'impiegata

e mio padre e mia madre, una sola

fanno crescere Luca, il mio unico amore

a volte penso che sia finita

ma è proprio allora che comincia la salita

Che fantastica storia è la vita

Che fantastica storia è la vita

E quando pensi che sia finita

E proprio allora che comincia la salita
Che fantastica storia è la vita

Mi chiamano Gesù e faccio il pescatore,

e del mare e del pesce sento ancora $1^{\prime}$

di mio padre e mia madre, su questa

nelle notti d'estate, sento ancora la

E quando penso che sia finita,

è proprio allora che comincia la salita.

Che fantastica storia è la vita.

Che fantastica storia è la vita.

Mi chiamo Aicha, come una canzone,

sono la quarta di

persone,

su questo scoglio di buona

scelgo la vita, l'unica salva.

E quando penso che sia finita,

è proprio adesso che comincia la salita.

Che fantastica storia è la vita.

Che fantastica storia è la vita-

\section{Nel testo della canzone ci sono delle parole annerite. Aiutati della canzone per indicare il sinonimo:}

\begin{tabular}{lll}
\hline 1) Ho sfidato (sfidare) & a) provocare & b) temere \\
\hline 2) Salita & a) discesa & b) ascesa \\
3) Scoglio & a) difficoltà & b) facilità \\
4) Scelgo (scegliere) & a) eleggere & b) scartare
\end{tabular}


(C) Italiano LinguaDue 2. 2021. H. Tolentino Quiñones, Uso della can₹one nella classe di lingua e cultura per sviluppare la competenza socioculturale

7. Leggi le seguenti parti parte dal testo della canzone. Cosa voleva dire il cantautore?

Mi chiamo Antonio e faccio il cantautore e mio padre e mia madre mi volevano dottore

ho sfidato il destino per la prima canzone

ho lasciato gli amici, ho perduto l'amore
Mi chiamo Aicha, come una canzone, sono la quarta di tremila persone,

su questo scoglio di buona speranza,

scelgo la vita, l'unica salva.

\section{Scegli un personaggio della canzone e lavora insieme a un compagno.} Uno di voi fa un'intervista al personaggio scelto.

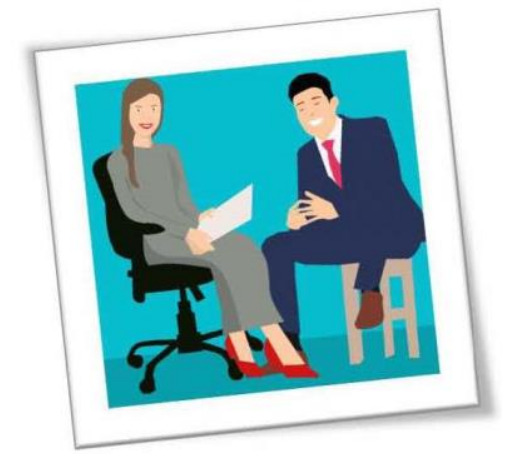

9. E per te, perchê è fantastica la vita? Scrivi un breve testo.

\section{PENSA}

\section{FABRIZIO MORO}

\section{https://www.youtube.com/watch?v=PaSU8hrgPYQ}

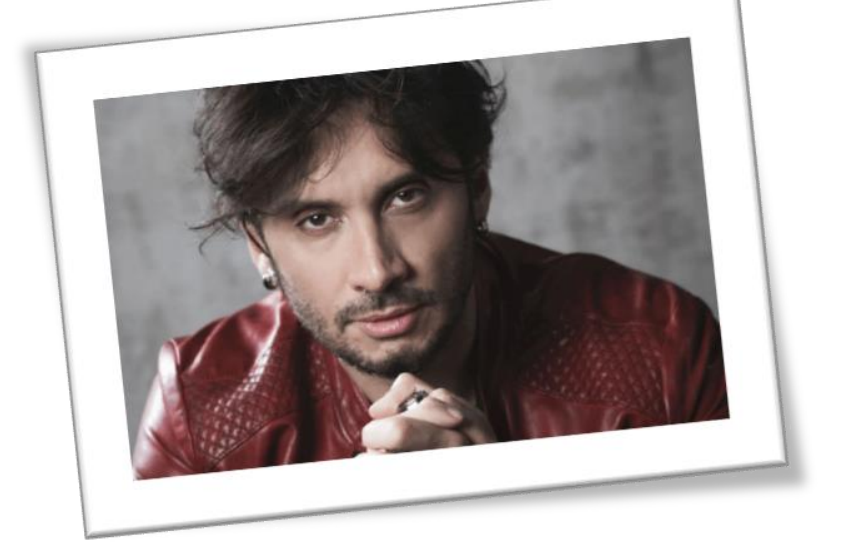


Fabrizio Moro, il cui vero nome è Fabrizio Mobrici, nasce il 9 aprile del 1975 a Roma, nel quartiere periferico di San Basilio, in una famiglia di origini calabresi. Dopo essersi iscritto all'istituto per la cinematografia e la televisione "Roberto Rossellini", si trasferisce a Setteville di Guidonia con il resto della famiglia, prima di fermarsi a Sant'Angelo Romano.

Musicista autodidatta, impara a suonare la chitarra da solo e scrive la sua prima canzone a quindici anni. Esibendosi in pub e locali con varie band, si fa apprezzare per le sue cover di brani degli U2 e dei Doors, prima di pubblicare il suo primo singolo, intitolato "Per tutta un'altra destinazione", nel 1996.

\section{Pensa}

Ci sono stati uomini che hanno scritto pagine

Appunti di una vita dal valore inestimabile

Insostituibili perché hanno denunciato

Il più corrotto dei sistemi troppo spesso ignorato

Uomini o angeli mandati sulla terra per combattere una guerra

Di faide e di famiglie sparse come tante biglie

Su un'isola di sangue che fra tante meraviglie

Fra limoni e fra conchiglie, massacra figli e figlie

Di una generazione costretta a non guardare

A parlare a bassa voce, a spegnere la luce

A commentare in pace ogni pallottola nell'aria

Ogni cadavere in un fosso

Ci sono stati uomini che passo dopo passo

Hanno lasciato un segno con coraggio e con impegno

Con dedizione contro un'istituzione organizzata

Cosa Nostra, cosa vostra, cos'è vostro?

È nostra, la libertà di dire

Che gli occhi sono fatti per guardare

La bocca per parlare, le orecchie ascoltano

Non solo musica, non solo musica

La testa si gira e aggiusta la mira, ragiona

A volte condanna, a volte perdona

Semplicemente

Pensa prima di sparare

Pensa prima di dire e di giudicare, prova a pensare

Pensa che puoi decidere tu

Resta un attimo soltanto, un attimo di più

Con la testa fra le mani

Ci sono stati uomini che sono morti giovani

Ma consapevoli che le loro idee

Sarebbero rimaste nei secoli come parole iperbole

Intatte e reali come piccoli miracoli

Idee di uguaglianza, idee di educazione

Contro ogni uomo che eserciti oppressione 
(C) Italiano LinguaDue 2. 2021. H. Tolentino Quiñones, Uso della can₹one nella classe di lingua e cultura per sviluppare la competenza socioculturale

Contro ogni suo simile, contro chi è più debole

Contro chi sotterra la coscienza nel cemento

Pensa prima di sparare

Pensa prima di dire e di giudicare, prova a pensare

Pensa che puoi decidere tu

Resta un attimo soltanto, un attimo di più

Con la testa fra le mani

Ci sono stati uomini che hanno continuato

Nonostante intorno fosse tutto bruciato

Perché in fondo questa vita non ha significato

Se hai paura di una bomba o di un fucile puntato

Gli uomini passano e passa una canzone

Ma nessuno potrà fermare mai la convinzione

Che la giustizia no, non è solo un'illusione

Pensa prima di sparare

Pensa prima di dire e di giudicare, prova a pensare

Pensa che puoi decidere tu

Resta un attimo soltanto, un attimo di più

Con la testa fra le mani

Pensa

Pensa che puoi decidere tu

Resta un attimo soltanto, un attimo di più

Con la testa fra le mani

\section{Osserva l'immagine, a cosa ti fa pensare?}
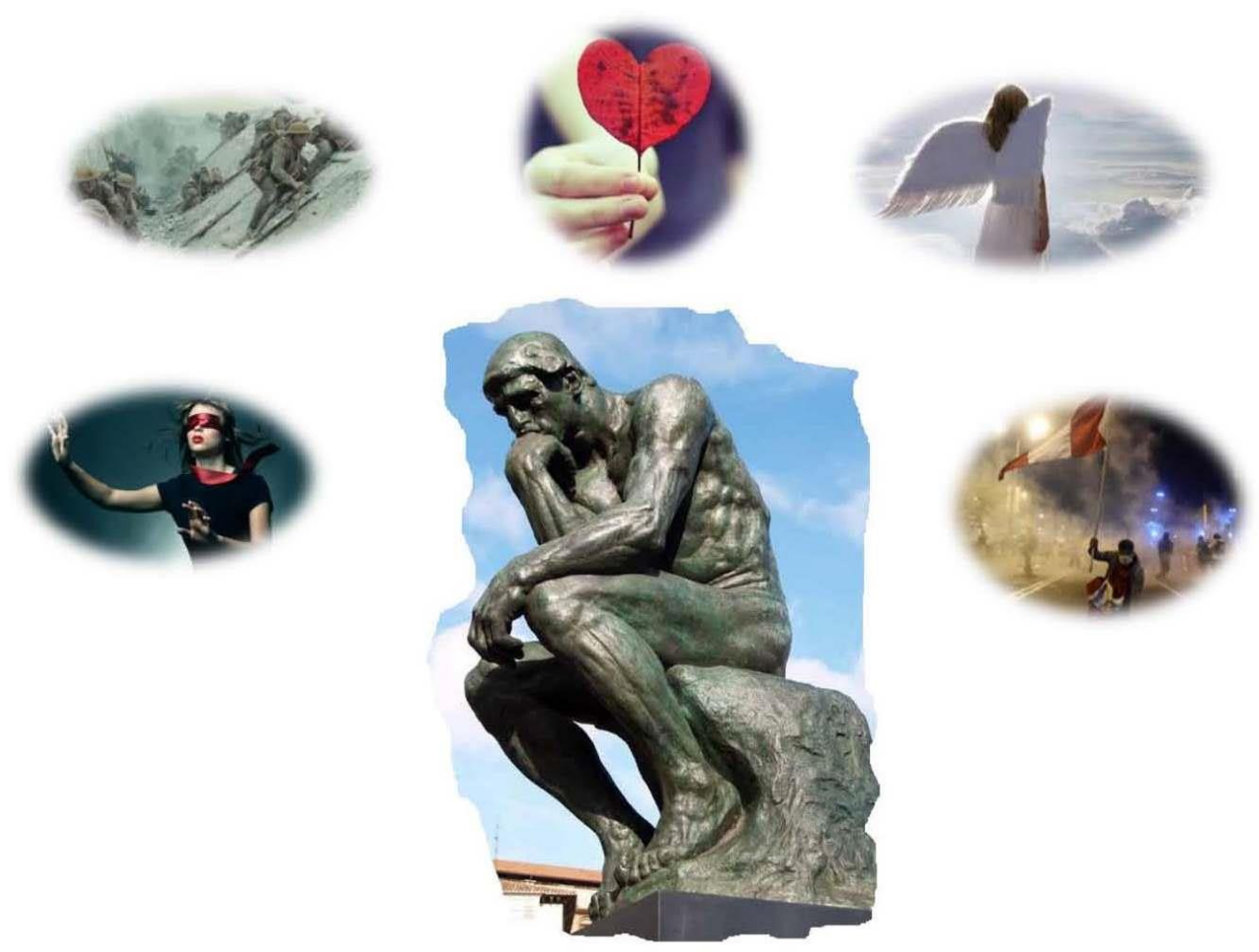
(C) Italiano LinguaDue 2. 2021. H. Tolentino Quiñones, Uso della canzone nella classe di lingua e cultura per sviluppare la competenza socioculturale

\section{Forma delle frasi, dopo ascolta per verificare se le tue frasi sono corrette.}
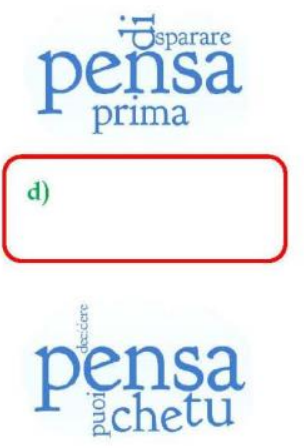

f)

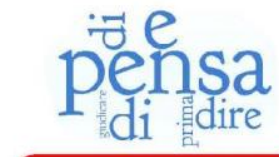

c)

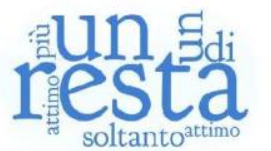

e)

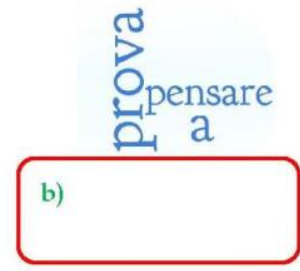

testąo

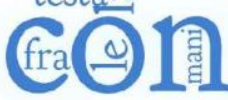

a)

\section{Ascolta la canzone Pensa di Fabrizio Moro.}

Ci sono stati uomini che hanno scritto pagine

Appunti di una vita dal valore inestimabile

Insostituibili perché hanno denunciato

Il più dei sistemi troppo

spesso ignorato

Uomini o angeli mandati sulla terra per una guerra

Di faide e di famiglie sparse come tante biglie

Su un'isola di sangue che fra tante meraviglie

Fra limoni e fra conchiglie... massacra figli e figlie

Di una costretta a

non guardare

A parlare a bassa voce a spegnere la luce

A commentare in ogni pallottola nell'aria

Ogni cadavere in un fosso

Ci sono stati uomini che passo dopo passo

Hanno lasciato un segno con e con impegno

Con dedizione contro un'istituzione organizzata

Cosa nostra... cosa vostra... cos'è vostro? è nostra... la libertà di dire

Che gli occhi sono fatti per guardare le orecchie Pensa prima di dire e di giudicare prova a La bocca per ascoltano...

Non solo musica non solo musica La testa si gira e aggiusta la mira

A volte condanna a volte perdona Semplicemente

Pensa prima di sparare

Pensa prima di dire e di giudicare prova a pensare

Pensa che puoi decidere tu

Resta un attimo soltanto un attimo di più

Con la testa fra le mani

Ci sono stati uomini che sono morti
Ma consapevoli che le loro idee Sarebbero rimaste nei secoli come parole iperbole

Intatte e reali come piccoli miracoli

Idee di uguaglianza idee di educazione Contro ogni uomo che eserciti

Contro ogni suo simile contro chi è più debole

Contro chi sotterra la coscienza nel cemento Pensa prima di sparare

Pensa prima di dire e di giudicare prova a

pensare

Pensa che puoi decidere tu

Resta un attimo soltanto un attimo di più Con la testa fra le mani

$\mathrm{Ci}$ sono stati uomini che hanno continuato Nonostante intorno fosse tutto bruciato Perché in fondo questa vita non ha significato

Se hai di una bomba o di

un fucile puntato

Gli uomini passano e passa una canzone

Ma nessuno potrà fermare mai la convinzione

Che la no... non è solo un'illusione Pensa prima di sparar pensare

Pensa che puoi decidere tu Resta un attimo soltanto un attimo di più Con la testa fra le mani Pensa. 
(C) Italiano LinguaDue 2. 2021. H. Tolentino Quiñones, Uso della canzone nella classe di lingua e cultura per sviluppare la competenza socioculturale

4. Leggi il testo della canzone e seleziona le parole "positive" e quelle "negative. Dopo trascrivili nei box.

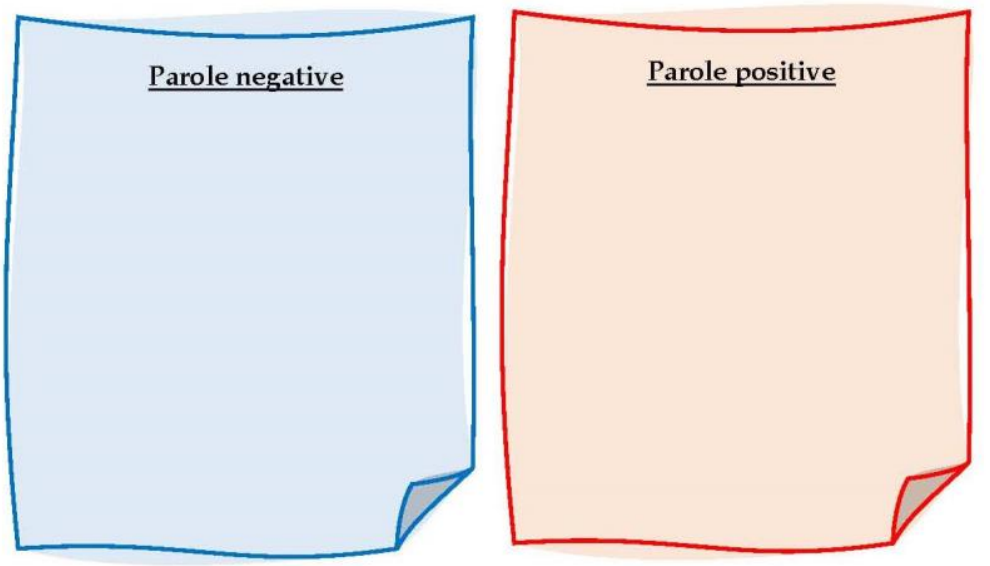

5. Trova le parole annerite nel testo della canzone e scrivile accanto al significato giusto.

\begin{tabular}{|l|l|}
\hline $\mathbf{1 .}$ & Involucro che protegge il corpo dei molluschi. \\
\hline $\mathbf{2 .}$ & Buca lunga e profonda, naturale o artificiale. \\
\hline $\mathbf{3 .}$ & $\begin{array}{l}\text { Nel puntare un'arma, linea ideale che unisce l'arma } \\
\text { stessa al bersaglio da colpire }\end{array}$ \\
\hline $\mathbf{4 .}$ & Sorta di guerra privata tra gruppi, spec. Familiari. \\
\hline $\mathbf{5 .}$ & Proiettili lanciati dalle armi da fuoco portatili. \\
\hline 6. & $\begin{array}{l}\text { Dedicarsi completamente e spontaneamente a una } \\
\text { persona, a una cosa, a un ideale. }\end{array}$ \\
\hline 7. & Palla di avorio per il gioco del biliardo. \\
\hline 8. Iperbole & $\begin{array}{l}\text { Figura retorica consistente nell'esagerare per } \\
\text { eccesso o per difetto. Amplificazione, enfatizzazione. }\end{array}$ \\
\hline
\end{tabular}

6. Cosa vuole dire il ritornello della canzone? Prova a dare una spiegazione usando un esempio.

Pensa prima di sparare

pensa prima di dire e di giudicare prova a pensare

pensa che puoi decidere tu

resta un attimo soltanto un attimo di più

con la testa fra le mani 
(C) Italiano LinguaDue 2. 2021. H. Tolentino Quiñones, Uso della canzone nella classe di lingua e cultura per sviluppare la competenza socioculturale

\section{Leggi il testo della canzone e rispondi alle seguenti domande.}

a) Che tipi di uomini ci sono stati?

b) Cosa fa la testa?

c) Perché la canzone si chiama Pensa?

8. Conosci qualche persona che ha lasciato segno nella storia dell'umanità? Lavora con un compagno, uno è il personaggio e l'altro un giornalista. Elaborate un'intervista.

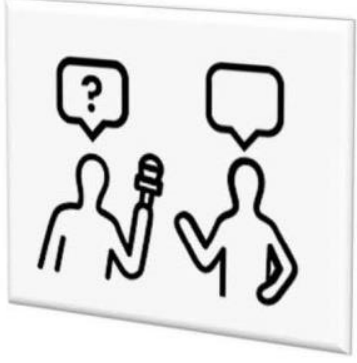

